\title{
THE COTANGENT COMPLEX OF A MORPHISM
}

\author{
BY \\ S. LICHTENBAUM AND M. SCHLESSINGER
}

0. Introduction. Let $A \rightarrow B \rightarrow C$ be a sequence of ring homomorphisms. (Unless otherwise noted all rings will be commutative with unit, and all modules and homomorphisms will be unitary.) Let $\Omega_{B / A}, \Omega_{C / A}, \Omega_{C / B}$ denote the respective modules of Kähler differentials (\$1.1). Then one obtains easily the following exact sequence of $C$-modules:

$$
\Omega_{B / A} \otimes_{B} C \rightarrow \Omega_{C / A} \rightarrow \Omega_{C / B} \rightarrow 0 .
$$

Also if $C=B \mid I$, where $I$ is an ideal in $B$, then $\Omega_{C / B}=0$ and one obtains the exact sequence

$$
I / I^{2} \stackrel{\bar{d}}{\longrightarrow} \Omega_{B / A} \otimes_{B} C \longrightarrow \Omega_{C / A} \longrightarrow 0
$$

where $\bar{d}$ is induced from the universal derivation $d$ of $B$ into $\Omega_{B / A}(\S 1.1)$.

In $\S 2$ we show that $(0.1)$ and $(0.2)$ are parts of a nine term exact sequence. To be precise, let $M$ be a $C$-module. Then we can form two exact sequences

$$
\begin{gathered}
\Omega_{B / A} \otimes_{B} M \rightarrow \Omega_{C / A} \otimes_{C} M \rightarrow \Omega_{C / B} \otimes_{C} M \rightarrow 0, \\
0 \rightarrow \operatorname{Der}_{B}(C, M) \rightarrow \operatorname{Der}_{A}(C, M) \rightarrow \operatorname{Der}_{A}(B, M)
\end{gathered}
$$

(see $\S 1.1$ for Der). We define functors $T_{i}(B \mid A, M)$ and $T^{i}(B \mid A, M) i=0,1,2$ (for any $B$-module $M$ ) such that $T_{0}(B \mid A, M)=\Omega_{B / A} \otimes_{B} M$ and $T^{0}(B \mid A, M)=\operatorname{Der}_{A}(B, M)$, and the $T_{i}$ (resp. $T^{i}$ ) fit into nine term exact sequences extending (0.3) (resp. $(0.4)$ ). The groups $T_{i}$ and $T^{i}$ are formed by taking homology and cohomology of a three term complex, the Cotangent Complex of $B$ over $A$. Also, under suitable finiteness conditions, the vanishing of the functor $T^{1}(B \mid A, \cdot)\left(\right.$ resp. $\left.T_{1}(B \mid A, \cdot)\right)$ is equivalent to $B$ being "smooth" (formerly "simple") over $A$, and the vanishing of $T^{2}(B \mid A, \cdot)$ (resp. $T_{2}(B \mid A, \cdot)$ is equivalent to $B$ being a "locally complete intersection over $A$." These and other vanishing criteria are discussed in \$3. The Jacobian criterion for nonsingularity of a variety is obtained as a natural consequence of these criteria. In $\S 4$ we apply the functors $T^{i}$ to the study of infinitesimal deformations, and obstructions thereto. In $\S 5$ we explain the role of the $T_{i}$ in a reformulation of the Grothendieck-Riemann-Roch Theorem.

Many other authors have studied, in many different guises, the homology and cohomology theories of commutative algebras, and have obtained some of the results which we prove in this paper. However, since our definitions are not the same (although in some cases equivalent to) those of previous authors, we have

Received by the editors December 16, 1965. 
tried to make this paper as self-contained as possible, and so we have included some proofs of known results. A brief historical sketch follows.

The functors $T_{0}$ and $T_{1}$ were first considered together in a Bourbaki report by Cartier, who treated only the case of field extensions [2]. In this report, he obtained the equality

$$
\operatorname{tr} \operatorname{deg} L / K=\operatorname{dim} \Omega_{L / K}-\operatorname{dim} T_{1}(L / K, L),
$$

which we prove in $\S 3.4$. He also showed that $T_{1}(L / K, L)=0$ if and only if $L$ is a separable extension of $K$. (The corresponding result for $T^{1}$ is due to Gerstenhaber [3].)

The work of Cartier was partially extended to commutative rings by Nakai, who obtained some segments of the change-of-rings exact sequence for homology (Propositions 1 and 9 of [12]). Grothendieck has demonstrated the existence of a six-term exact sequence in cohomology in [5, Chapter IV]. (Note that Grothendieck defines $T^{1}$ in terms of commutative algebra extensions, and his Exal$\operatorname{com}_{A}(B, M)$ is our $T^{1}(B / A, M)$.) He also has analogous results for noncommutative and topological rings, and the vanishing criteria for $T^{1}$. In unpublished work, Grothendieck has used a two-term cotangent complex to define $T^{1}$ and $T_{1}$ to get a six-term exact sequence for both the homological and cohomological functors.

Cohomology groups $H^{i}(B / A, M), 0 \leqq i<\infty$, are defined by Shukla in [15] where $B$ is an arbitrary associative $A$-algebra, and by Harrison [7] for the case when $B$ is commutative and $A$ is a field. The results of $\S 4$ indicate that our cohomology theory is essentially a "commutativization" of Shukla's, except for a shift in dimension. Our $T^{i}$ is Harrison's $H^{i+1}$ for $i=0,1$ (when $A$ is a field). Harrison also obtained a nine-term exact sequence for change of rings, except that three of his terms cannot be interpreted as cohomology groups because of his requirement that the ground ring be a field.

Gerstenhaber has recently found a general method of defining cohomology theories (in dimension $\leqq 3$ ) in arbitrarily equationally defined categories [16]. (Note that because of a difference in notation, his range of definition is the same as ours.) In the category of commutative rings, Gerstenhaber's "correct" $H_{A}^{i+1}(B, M)$ is the same as our $T^{i}(B / A, M), i=0,1,2$, but Gerstenhaber does not obtain the change of rings exact sequence. (It would be interesting to know if this is valid in an arbitrary equationally defined category.) Gerstenhaber also gives in [16] a general definition of two-term extension which reduces to our definition (2.1.1) in the commutative case.

For applications of the functor $T_{1}$ to the definition of the "canonical class" for algebraic varieties over nonperfect ground fields and its relation to the work of Kunz [9], see [10]. For further applications of the functors $T^{1}$ and $T^{2}$ to the study of infinitesimal deformations and "formal moduli," see [13].

We wish to express our appreciation to John Tate for many interesting discussions and much encouragement, and in particular for pointing out to each of us that we were working on the same problem. 


\section{Preliminaries.}

1.0. Conventions. The conventions of commutative algebra, mentioned in the first paragraph of the introduction, remain in force except in $\$ 4.2 .3$, where we have reference to a commutative $A$-algebra $J$ without unit. $A, B$, and $C$ always denote rings.

1.1. The module of differentials. If $A \rightarrow B$ is a ring homomorphism and $M$ is a $B$-module, let $\operatorname{Der}_{A}(B, M)$ denote the module of $A$-derivations of $B$ into $M$, i.e., $A$-linear maps $D$ of $B$ into $M$ such that $D(x y)=x D y+y D x$ for $x$ and $y$ in $B$. The module of Kähler differentials of $B$ over $A$, denoted $\Omega_{B \mid A}$, represents the functor $M \rightarrow \operatorname{Der}_{A}(B, M)$. That is, there is a universal $A$-derivation $d: B \rightarrow \Omega_{B / A}$ which induces a bijection

$$
d^{*}: \operatorname{Hom}_{B}\left(\Omega_{B / A}, M\right) \cong \operatorname{Der}_{A}(B, M)
$$

for each $B$-module $M \cdot\left(d^{*}(f)=f \circ d\right)$.

Clearly $\Omega_{B / A}$ may be obtained by forming the free $B$-module on the symbols $d x(x \in B)$ and dividing out by the relations $d\left(a x+a^{\prime} y\right)-a \cdot d x-a^{\prime} \cdot d y$ and $d(x y)$ $-x \cdot d y-y \cdot d x$ for $a, a^{\prime} \in A$ and $x, y \in B$. The exact sequences of the introduction now follow easily from the fact that $\Omega_{B / A}$ represents the derivation functor. In a similar way one proves that if $A \rightarrow A^{\prime}$ is a ring homomorphism and if $B^{\prime}=B \otimes_{A} A^{\prime}$ then we get a bijection

$$
\Omega_{B \mid A} \otimes_{A} A^{\prime} \cong \Omega_{B^{\prime} \mid A^{\prime}}
$$

2. The cotangent complex.

\subsection{Basic definitions.}

2.1.1. Definition. Let $A \rightarrow B$ be a ring homomorphism. By a (two-term) extension of $B$ over $A$ we mean an exact sequence

$$
(\mathscr{E}): 0 \longrightarrow E_{2} \longrightarrow E_{1} \stackrel{e_{2}}{\longrightarrow} R \stackrel{e_{1}}{\longrightarrow} B \stackrel{e_{0}}{\longrightarrow} 0
$$

where $e_{0}$ is a surjection of $A$-algebras, $e_{2}$ and $e_{1}$ are homomorphisms of $R$-modules, and

$$
e_{1}(x) y=e_{1}(y) x
$$

for $x, y \in E_{1}$. Notice that if $I=\operatorname{Ker} e_{0}$, then $I E_{2}=0$, so that $E_{2}$ is a B-module. In fact, if $a \in I$ and $x \in E_{2}$, choose $y \in E_{1}$ such that $e_{1}(y)=a$. Then $e_{2}(a x)=e_{1}(y) e_{2}(x)$ $=e_{1} e_{2}(x) \cdot y=0$, so $a x=0$.

Let $A^{\prime}$ be an $A$-algebra, $B^{\prime}$ an $A^{\prime}$-algebra, and $\mathscr{E}^{\prime}$ an extension of $B^{\prime}$ over $A^{\prime}$. By a homomorphism $\alpha: \mathscr{E} \rightarrow \mathscr{E}^{\prime}$ of extensions we mean a collection $\left(b, \alpha_{0}, \alpha_{1}, \alpha_{2}\right)$ of maps which render the diagram

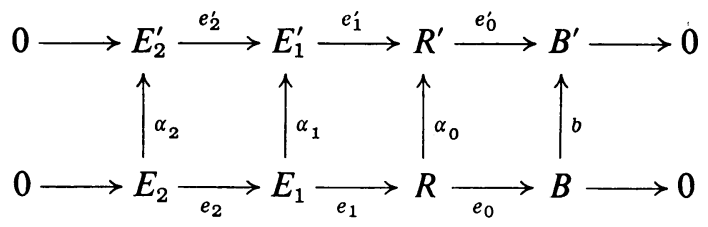


commutative. Here, $b$ and $\alpha_{0}$ are homomorphisms of $A$-algebras, and $\alpha_{1}$ and $\alpha_{2}$ are homomorphisms of $R$-modules (where we regard $R^{\prime}$ and $B^{\prime}$ as $A$-algebras via $A \rightarrow A^{\prime}$, and $E_{1}^{\prime}$ and $E_{2}^{\prime}$ as $R$-algebras via $\alpha_{0}$ ).

2.1.2. Definition. With each extension $\mathscr{E}$ of $B$ over $A$ we associate a three term complex $L \cdot(\mathscr{E})$ of $B$ modules:

$$
L \cdot(\mathscr{E}): 0 \longrightarrow E_{2} \stackrel{d_{2}}{\longrightarrow} E_{1} \otimes_{R} B \stackrel{d_{1}}{\longrightarrow} \Omega_{R / A} \otimes_{R} B \longrightarrow 0 .
$$

Here $\Omega_{R / A}$ is the module of Kähler differentials, $d_{2}$ is induced from $e_{2}$, and $d_{1}$ is defined as follows: let $I=\operatorname{Ker} e_{0}$. Then $\operatorname{Im} e_{1}=I$ and we have $d: I / I^{2} \rightarrow \Omega_{R / A} \otimes_{R} B$ (§1.1). Put $d_{1}=d \circ\left(e_{1} \otimes_{R} B\right)$.

Extensions of $B$ over $A$ are all obtained in the following way. Choose a surjection $e_{0}: R \rightarrow B$ of $A$-algebras, and let $I=\operatorname{Ker} e_{0}$. Then choose an exact sequence

$$
0 \longrightarrow U \stackrel{i}{\longrightarrow} F \stackrel{j}{\longrightarrow} I \longrightarrow 0
$$

of $R$ modules and define $\varphi: F \otimes F \rightarrow F$ by the formula

$$
\varphi(x \otimes y)=j(x) y-j(y) x .
$$

Let $U_{0} \subseteq U$ be the image of $\varphi$, and let $e_{2}: U / U_{0} \rightarrow F / U_{0}$, and $e_{1}: F / U_{0} \rightarrow R$ be the induced maps.

2.1.3. Definition. An extension of the form

$$
0 \rightarrow U / U_{0} \rightarrow F / U_{0} \rightarrow R \rightarrow B \rightarrow 0
$$

where $R$ is a polynomial algebra over $A$ and $F$ is a free $R$-module is called a free extension of $B$ over $A$.

It is clear that if

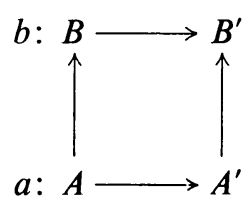

is a commutative diagram of ring homomorphisms, and $\mathscr{E}$ (resp. $\mathscr{E}^{\prime}$ ) is a free (resp. arbitrary) extension of $B$ over $A$, then there exists a homomorphism $\alpha: \mathscr{E} \rightarrow \mathscr{E}^{\prime}$ extending $b$, and hence a homomorphism $\bar{\alpha}: L \cdot(\mathscr{E}) \otimes_{B} B^{\prime} \rightarrow L \cdot\left(\mathscr{E}^{\prime}\right)$.

A complex of the form $L \cdot(\mathscr{E})$, where $E$ is a free extension of $B$ over $A$ is called a cotangent complex of $B$ over $A$. The fact that polynomial rings possess the following "lifting" property implies that any two cotangent complexes are homotopic (2.1.6).

2.1.4. Definition. We say that an $A$-algebra $R$ has property (L) if the following is true. Let $S$ be an $A$-algebra and $u: M \rightarrow S$ a homomorphism of $S$-modules such that $u(x) y=u(y) x$, for $x, y \in M$. Then for any pair $f, g: R \rightarrow S$ of $A$-homomor- 
phisms such that $\operatorname{Im}(f-g) \subseteq \operatorname{Im}(u)$, there exists a biderivation $\lambda: R \rightarrow M$ such that $u \circ \lambda=f-g$.

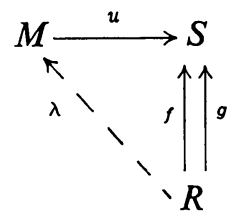

( $S$ and $M$ are bi- $R$-modules via $f$ and $g$. A biderivation in this case is an $A$-linear map $\lambda$ such that $\lambda(x y)=f(x) \lambda(y)+g(y) \lambda(x)$. Observe that $f-g$ is a biderivation.)

2.1.5. LemMA. Given a commutative diagram (*) of ring homomorphisms, let $\mathscr{E}$ (resp. $\left.\mathscr{E}^{\prime}\right)$ be an extension of $B$ over $A$ (resp. $B^{\prime}$ over $A^{\prime}$ ) and let $\alpha, \beta: \mathscr{E} \rightarrow \mathscr{E}^{\prime}$ be a pair of homomorphisms, both extending $b$.

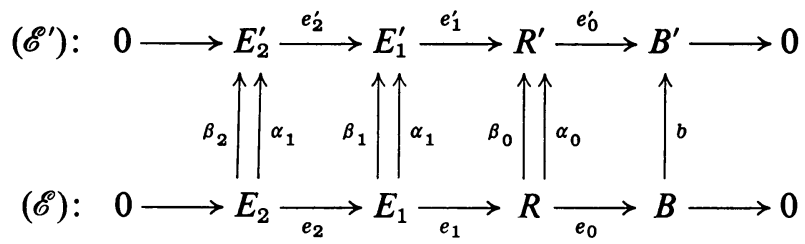

Then if $R$ has property (L), $\bar{\alpha}$ and $\bar{\beta}$ are homotopic maps of $L \cdot(\mathscr{E}) \otimes_{B} B^{\prime}$ to $L \cdot(\mathscr{E})$.

Proof. By property (L) with $R=R, S=R^{\prime}, M=E_{1}^{\prime}$, and $u=e_{1}^{\prime}, f=\alpha_{0}$ and $g=\beta_{0}$, there exists a biderivation $\lambda: R \rightarrow E_{1}^{\prime}$ such that $e_{1}^{\prime} \circ \lambda=\alpha_{0}-\beta_{0}$. Let $\theta: E_{1} \rightarrow E_{1}^{\prime}$ be the ( $A$-linear) map defined by $\theta=\beta_{1}-\alpha_{1}+\lambda \circ e_{1}$. We claim first that $e_{1}^{\prime} \circ \theta=0$. In fact, $e_{1}^{\prime} \circ \theta=\left(\beta_{0}-\alpha_{0}+e_{1}^{\prime} \circ \lambda\right) \circ e_{1}=0$. Thus $\operatorname{Im} \theta$ is included in the $B^{\prime}$ module $\operatorname{Im} e_{2}^{\prime}$ on which the actions of $R$ via $\alpha_{0}$ and $\beta_{0}$ agree. We claim next that $\theta$ is actually $R$-linear, i.e., $\theta(r x)=\alpha_{0}(r) \theta(x)$ for $r \in R, x \in E_{1}$. On the one hand,

$$
\begin{aligned}
\theta(r x) & =\beta_{0}(r) \beta_{1}(x)-\alpha_{0}(r) \alpha_{1}(x)+\lambda\left(r \cdot e_{1}(x)\right) \\
& =\beta_{0}(r) \beta_{1}(x)-\alpha_{0}(r) \alpha_{1}(x)+\alpha_{0}(r) \lambda\left(e_{1}(x)\right)+\beta_{0}\left(e_{1}(x)\right) \lambda(r),
\end{aligned}
$$

and on the other hand,

$$
\alpha_{0}(r) \theta(x)=\alpha_{0}(r) \beta_{1}(x)-\alpha_{0}(r) \alpha_{1}(x)+\alpha_{0}(r) \lambda\left(e_{1}(x)\right) .
$$

Thus

$$
\begin{aligned}
\theta(r x)-\alpha_{0}(r) \theta(x) & =\left(\beta_{0}(r)-\alpha_{0}(r)\right) \beta_{1}(x)+\beta_{0}\left(e_{1}(x)\right) \lambda(r) \\
& =-e_{1}^{\prime}(\lambda(r)) \beta_{1}(x)+e_{1}^{\prime}\left(\beta_{1}(x)\right) \lambda(r)=0
\end{aligned}
$$

and $\theta$ is $R$-linear.

Now the biderivation $\lambda: R \rightarrow E_{1}^{\prime}$ induces a derivation $\bar{\lambda}: R \rightarrow E_{1}^{\prime} \otimes_{R^{\prime}} B^{\prime}=L^{1}\left(\mathscr{E}^{\prime}\right)$, which, by the universal mapping property of $\Omega_{R / A}$ induces $\bar{\lambda}_{0}: \Omega_{R / A} \rightarrow L^{1}\left(\mathscr{E}^{\prime}\right)$, or what is the same thing, $\lambda_{0}: \Omega_{R / A} \otimes_{R} B^{\prime}=L^{0}(\mathscr{E}) \bigotimes_{B} B^{\prime} \rightarrow L^{1}\left(\mathscr{E}^{\prime}\right)$. The $R$-linear map $\theta: E_{1} \rightarrow \operatorname{Im} e_{2}^{\prime}$ induces $\lambda_{1}: L^{1}(\mathscr{E}) \otimes_{B} B^{\prime} \rightarrow L^{2}\left(\mathscr{E}^{\prime}\right)$. Finally, if $d$ (resp. $d^{\prime}$ ) denotes differentiation in $L(\mathscr{E}) \otimes B^{\prime}$ (resp. $L\left(\mathscr{E}^{\prime}\right)$ ) then it follows easily from the definitions 
of $\lambda$ and $\theta$ that $d_{1}^{\prime} \circ \lambda_{0}=\bar{\alpha}_{0}-\bar{\beta}_{0}, \lambda_{0} \circ d_{1}+d_{2}^{\prime} \circ \lambda_{1}=\bar{\alpha}_{1}-\bar{\beta}_{1}$ and $\lambda_{1} \circ d_{2}=\bar{\alpha}_{2}-\bar{\beta}_{2}$. Thus $\left(\lambda_{0}, \lambda_{1}\right)$ is the desired homotopy operator.

2.1.6. LeMmA. If $R$ is a polynomial algebra over $A$, then $R$ has property (L) of Definition 2.1.4.

Proof. Let $u: M \rightarrow S$, and $f, g: R \rightarrow S$ be given as in the definition, and suppose $R=A\left[T_{i}\right]_{i \in I}$ (I is an index set). Since $\operatorname{Im}(f-g) \subseteq \operatorname{Im}(u)$, there exist elements $\lambda\left(T_{i}\right)$ in $M$ such that $u\left(\lambda\left(T_{i}\right)\right)=f\left(T_{i}\right)-g\left(T_{i}\right)$. Define $\lambda$ on monomials $T_{i_{1}} \cdots T_{i_{n}}$ by the formula

$$
\lambda\left(T_{i_{1}} \cdots T_{i_{n}}\right)=\sum_{k=1}^{n} f\left(T_{i_{1}} \cdots T_{i_{k-1}}\right) \lambda\left(T_{i_{k}}\right) g\left(T_{i_{k+1}} \cdots T_{i_{n}}\right) .
$$

To see that $\lambda$ is well defined, we show that the right-hand side of $(*)$ remains invariant under transpositions $\left(i_{p}, i_{p+1}\right)$ of the indices.

$$
\begin{aligned}
\lambda\left(T_{i_{1}} \cdots\right. & \left.T_{i_{p+1}} T_{i_{p}} \cdots T_{i_{n}}\right)-\lambda\left(T_{i_{1}} \cdots T_{i_{p}} T_{i_{p+1}} \cdots T_{i_{n}}\right) \\
= & f\left(T_{i_{1}} \cdots T_{i_{p-1}}\right) \lambda\left(T_{i_{p+1}}\right) g\left(T_{i_{p}} T_{i_{p+2}} \cdots T_{i_{n}}\right) \\
& +f\left(T_{i_{1}} \cdots T_{i_{p-1}} T_{i_{p+1}}\right) \lambda\left(T_{i_{p}}\right) g\left(T_{i_{p+2}} \cdots T_{i_{n}}\right) \\
& -f\left(T_{i_{1}} \cdots T_{i_{p-1}}\right) \lambda\left(T_{i_{p}}\right) g\left(T_{i_{p+1}} \cdots T_{i_{n}}\right) \\
& \quad-f\left(T_{i_{1}} \cdots T_{i_{p}}\right) \lambda\left(T_{i_{p+1}}\right) g\left(T_{i_{p+2}} \cdots T_{i_{n}}\right) \\
= & c(u(y) x-u(x) y)=0
\end{aligned}
$$

where $c=f\left(T_{i_{1}} \cdots T_{i_{p-1}}\right) g\left(T_{i_{p+2}} \cdots T_{i_{n}}\right), x=\lambda\left(T_{i_{p}}\right)$ and $y=\lambda\left(T_{i_{p+1}}\right)$.

If we extend $\lambda$ to all of $R$ by linearity, it follows easily from $(*)$ that $\lambda$ is a biderivation: $\lambda(x y)=f(x) \lambda(y)+g(y) \lambda(x)$, for $x$ and $y \in R$. Since $f-g$ is also a biderivation, and $u\left(\lambda\left(T_{i}\right)\right)=f\left(T_{i}\right)-g\left(T_{i}\right)$, we must have $u \circ \lambda=f-g$.

As a corollary we get

2.1.7. Proposition. Let

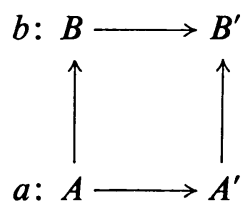

be a commutative diagram of ring homomorphisms, and $\mathscr{E}$ (resp. $\left.\mathscr{E}^{\prime}\right)$ an extension of $B$ over $A$ (resp. $B^{\prime}$ over $\left.A^{\prime}\right)$. If $\mathscr{E}$ is free, then:

(i) There exists a homomorphism $\alpha: \mathscr{E} \rightarrow \mathscr{E}^{\prime}$ extending $b$.

(ii) If $\beta: \mathscr{E} \rightarrow \mathscr{E}^{\prime}$ is any other homomorphism extending $b$, then $\bar{\alpha}$ and $\bar{\beta}$ are homotopic maps of $L \cdot(\mathscr{E}) \bigotimes_{B} B^{\prime}$ to $L^{\cdot}\left(\mathscr{E}^{\prime}\right)$.

2.1.8. Definition. If $B$ is an $A$-algebra, $\mathscr{E}$ is a free extension of $B$ over $A$, we call $L \cdot(\mathscr{E})$ a cotangent complex of $B$ over $A$.

2.1.9. Thus 2.1.7 implies in particular that any two cotangent complexes of $B$ over $A$ are homotopically equivalent. 
2.2. Functorial properties. The following two propositions establish the behavior of cotangent complexes under (flat) base extension and localization.

2.2.1. Let $B$ and $A^{\prime}$ be $A$-algebras, and $B^{\prime}=B \otimes_{A} A^{\prime}$ so that we have a product diagram

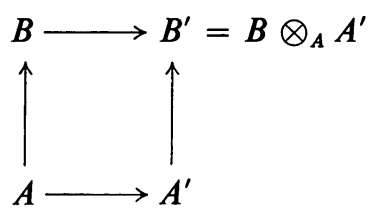

Suppose either that (i) $A^{\prime}$ is flat over $A$, or (ii) $B$ is flat over $A$, and let $\mathscr{E}$ be an extension of $B$ over $A$. Then

(a) $\mathscr{E}^{\prime}=\mathscr{E} \otimes_{A} A^{\prime}$ is an extension of $B^{\prime}$ over $A^{\prime}$.

(b) The natural homomorphism $\mathscr{E} \rightarrow \mathscr{E}^{\prime}$ induces an isomorphism $L \cdot(\mathscr{E}) \bigotimes_{B} B^{\prime}$ $\cong L \cdot\left(\mathscr{E}^{\prime}\right)$.

(c) If $\mathscr{E}$ is free, then so is $\mathscr{E}^{\prime}$. Thus if $L^{\cdot}(\mathscr{E})$ is a cotangent complex of $B$ over $A$, then $L \cdot(\mathscr{E}) \bigotimes_{B} B^{\prime}$ is a cotangent complex of $B^{\prime}$ over $A^{\prime}$.

Proof. We shall need the following observations:

(a) Given a ring homomorphism $R \rightarrow R^{\prime}$, and a homomorphism $u: F \rightarrow R$ of $R$ modules, define $\varphi: F \otimes_{R} F \rightarrow F$ by $\varphi(x \otimes y)=u(x) y-u(y) x$. If we put $F^{\prime}=F$ $\otimes_{R} R^{\prime}$, then the induced map $\varphi^{\prime}: F^{\prime} \otimes_{R^{\prime}} F^{\prime} \rightarrow F^{\prime}$ is given by $\varphi^{\prime}\left(x^{\prime} \otimes y^{\prime}\right)=u^{\prime}\left(x^{\prime}\right) y^{\prime}$ $-u^{\prime}\left(y^{\prime}\right) x^{\prime}$.

(b) In the diagram of 2.1.6, with $B^{\prime}=B \otimes_{A} A^{\prime}$,

$$
\Omega_{B / A} \otimes_{A} A^{\prime} \cong \Omega_{B^{\prime} \mid A^{\prime}}
$$

The proposition follows directly from these observations.

2.2.1 applies in particular when $A^{\prime}$ is of the form $A T^{-1}$, where $T$ is a multiplicative system in $A$. We conclude that the formation of cotangent complexes "respects localization downstairs." To get an analogous statement for localization in $B$ we use

2.2.2. Lemma. Let $R$ be an A-algebra, and $T$ a multiplicative system in $R$. If $R$ has property (L) of Definition 2.1.4, then so does $R T^{-1}$.

Proof. Consider the diagram of 2.1.4 for $R^{\prime}=R T^{-1}$.

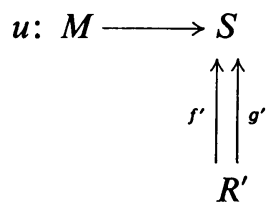

and let $f, g: R \rightarrow S$ be the homomorphisms induced from $R \rightarrow R^{\prime}$. Since $R$ has property (L), there exists a biderivation $\lambda: R \rightarrow M$ such that $u \circ \lambda=f-g$. Let 
$r / t \in R^{\prime}$ where $r \in R, t \in T$. Solving the equation $\lambda(r)=\lambda(t \cdot r / t)=\alpha(t) \lambda^{\prime}(r / t)+\beta(r / t) \lambda(t)$ for $\lambda^{\prime}$ we get

$$
\lambda^{\prime}(r / t)=[\beta(t) \lambda(r)-\beta(r) \lambda(t)] / \alpha(t) \beta(t) .
$$

Making liberal use of the relation $u(x) y=u(y) x$ for $x, y \in M$, one can show by a tedious but straightforward calculation that $(*)$ actually defines a biderivation $\lambda^{\prime}$ extending $\lambda$. Since $f^{\prime}-g^{\prime}$ is a biderivation extending $f-g$, it is clear that $\mu \circ \lambda^{\prime}$ $=f^{\prime}-g^{\prime}$.

2.2.3. Proposition. Let $B$ be an A-algebra, and $T$ a multiplicative system in $B$. If $L \cdot(\mathscr{E})($ resp. $L \cdot(\mathscr{F}))$ is a cotangent complex for $B\left(\right.$ resp. $\left.B T^{-1}\right)$ over $A$, then any homomorphism $\mathscr{E} \rightarrow \mathscr{F}$ (extending $B \rightarrow B T^{-1}$ ) induces a homotopy equivalence

$$
L \cdot(\mathscr{E}) \otimes_{B} B T^{-1} \rightarrow L \cdot(\mathscr{F}) .
$$

Proof. Let $\mathscr{E}: 0 \rightarrow E_{2} \rightarrow E_{1} \rightarrow R \rightarrow B \rightarrow 0$ be a free extension of $B$ over $A$. Let $U \subset R$ be the inverse image of $T$, and let $\mathscr{E}^{\prime}=\mathscr{E} \otimes_{R} R U^{-1}$. Since $R U^{-1}$ is flat over $R, \mathscr{E}^{\prime}$ is an extension of $B T^{-1}$ over $A$ (see observation (a) in the proof of 2.2.1). It is clear that the natural homomorphism $\mathscr{E} \rightarrow \mathscr{E}$ induces an isomorphism $L \cdot(\mathscr{E})$ $\otimes_{B} B T^{-1} \cong L \cdot\left(\mathscr{E}^{\prime}\right)$.

Now let $\mathscr{F}: 0 \rightarrow F_{2} \rightarrow F_{1} \rightarrow Q \rightarrow B T^{-1} \rightarrow 0$ be a free extension of $B T^{-1}$ over $A$, let $V \subset Q$ be the inverse image of $T$, and let $\mathscr{F}^{\prime}=\mathscr{F} \otimes_{Q} Q V^{-1}$. Then there exists a homomorphism $\alpha: \mathscr{F} \rightarrow \mathscr{E}^{\prime}$ extending the identity on $B T^{-1}$. Since $\alpha^{0}(V)$ $\subseteq U, \alpha$ induces $\alpha^{\prime}: \mathscr{F}^{\prime} \rightarrow \mathscr{E}^{\prime}$. On the other hand, $\beta: \mathscr{E} \rightarrow \mathscr{F}^{\prime}$ (extending $B \rightarrow B T^{-1}$ ) induces $\beta^{\prime}: \mathscr{E}^{\prime} \rightarrow \mathscr{F}^{\prime}$. Thus we have homomorphisms $\beta^{\prime}: \mathscr{E}^{\prime} \rightarrow \mathscr{F}^{\prime}$ and $\alpha^{\prime}: \mathscr{F}^{\prime} \rightarrow \mathscr{E}^{\prime}$ both extending the identity on $B T^{-1}$. Since $R U^{-1}$ and $Q V^{-1}$ have property (L) (2.2.2), $\bar{\alpha}^{\prime} \circ \bar{\beta}^{\prime}$ (resp. $\left.\bar{\beta}^{\prime} \circ \bar{\alpha}^{\prime}\right)$ is homotopic to the identity on $L \cdot\left(\mathscr{E}^{\prime}\right)$ (resp. $L \cdot(\mathscr{F})$ ). But $L^{\cdot}(\mathscr{E}) \otimes B T^{-1} \cong L^{\cdot}\left(\mathscr{E}^{\prime}\right)$ and $L^{\cdot}(\mathscr{F}) \rightarrow L^{\cdot}\left(\mathscr{F}^{\prime}\right)$ is a homotopy equivalence and we are done.

\section{The Change of Rings Exact Sequence.}

2.2.4. TheOREM. Let $A \rightarrow B \rightarrow C$ be a sequence of ring homomorphisms. Let $\mathscr{E}$ (resp. $\mathscr{G}$ ) be a free extension of $B$ over $A$ (resp. $C$ over $B$ ). Then there exists a free extension $\mathscr{F}$ of $C$ over $A$ and homomorphisms $\mathscr{E} \rightarrow \mathscr{F} \rightarrow \mathscr{G}$ extending $A \rightarrow B \rightarrow C$ such that the sequence

$$
0 \rightarrow L^{\cdot}(\mathscr{E}) \otimes_{B} C \rightarrow L^{\cdot}(\mathscr{F}) \rightarrow L^{\cdot}(\mathscr{G}) \rightarrow 0
$$

is exact, except that $L^{2}(\mathscr{E}) \bigotimes_{B} C \rightarrow L^{2}(\mathscr{F})$ need not be injective.

Proof. We have $(\mathscr{E}): 0 \rightarrow U / U_{0} \rightarrow F / U_{0} \rightarrow R \rightarrow B \rightarrow 0$ and $(\mathscr{G}): 0 \rightarrow W / W_{0}$ $\rightarrow H / W_{0} \rightarrow T \rightarrow C \rightarrow 0$, where $R=A\left[X_{i}\right], T=B\left[Y_{j}\right]$. Put $I=\operatorname{Ker}(R \rightarrow B)$ and $K=\operatorname{Ker}\left(T \rightarrow C\right.$ ). Now define $S=R\left[Y_{j}\right]=A\left[X_{i}, Y_{j}\right]$ (so that $S \otimes_{R} B \cong T$ ) and put $J=\operatorname{Ker}(S \rightarrow C)$.

Let $H^{*}$ be a free $S$-module such that $H^{*} \otimes_{S} T \cong H$ and define $G=F \otimes_{R} S \oplus H^{*}$. 
Define $u: G \rightarrow J$ as follows: $u \mid F \otimes_{R} S$ is the composition $F \otimes_{R} S \rightarrow I \otimes_{R} S \rightarrow J$, and $u / H^{*}$ is a lifting of $H^{*} \rightarrow K$ to $H^{*} \rightarrow J$. If we put $V=\operatorname{Ker}(G \rightarrow J)$ and $W^{*}=\operatorname{Ker}\left(H^{*} \rightarrow K\right)$ we get the following commutative diagram

$(*)$

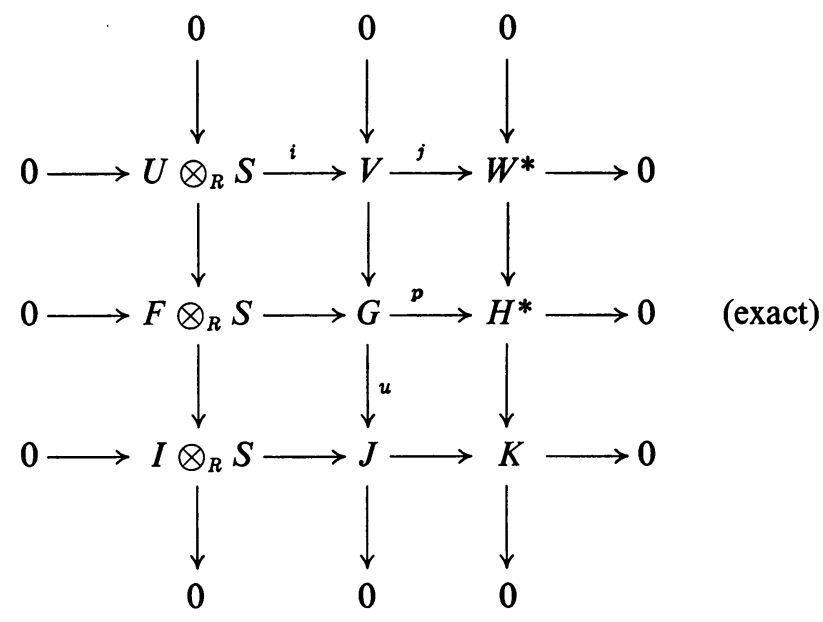

in which the rows and columns are exact. (Note that $S$ is flat over $R$, so that in particular $I \otimes_{R} S=I S$.) (*) induces a commutative diagram (**)

(**)

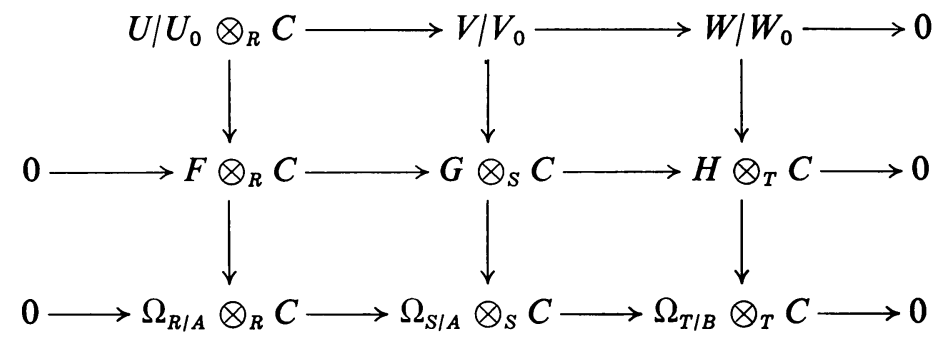

whose rows we must prove exact.

Reading from left to right on the bottom row of (**) we have free $C$-modules on generators $d X_{i}$, then $d X_{i}, d Y_{j}$ and finally $d Y_{j}$, thus the bottom row of $(* *)$ is exact. The middle row of $(* *)$ is exact since it is obtained from the middle row of (*) by applying $\otimes_{S} C$, and $H^{*}$ is free over $S$. Finally, it is clear that the composition $U / U_{0} \otimes_{R} C \rightarrow V / V_{0} \rightarrow W / W_{0}$ is zero, and that $V / V_{0} \rightarrow W / W_{0}$ is surjective. To prove the top row of $(* *)$ exact we must show that if $v \in V$ goes to 0 in $W$, then there exists $v_{0} \in V_{0}$ such that $v-v_{0}$ comes from $U \otimes_{R} S$. Since the top row of (*) is exact, $v-v_{0} \in \operatorname{Im}(i)$ if and only if $j\left(v-v_{0}\right)=0$. So we must prove that $j\left(V_{0}\right)$ $\supseteq \operatorname{Ker}\left(W^{*} \rightarrow W\right)$. But $\operatorname{Ker}\left(W^{*} \rightarrow W\right)=I H^{*} \cap W^{*}$, and we claim that $j\left(V_{0}\right) \supseteq I H^{*}$. In fact if $a \in I S=I \otimes_{R} S$, and $h \in H^{*}$, pick $x \in G, y \in H^{*}$ such that $u(x)=a$, $p(y)=h$. Then $z=u(x) y-u(y) z \in V_{0}$, and $p(x)=0$. Thus $j(z)=p(z)=a h$.

2.3. The cotangent functors. Let $B$ be an $A$-algebra, $\mathscr{E}$ a free extension of $B$ over $A$, and $M$ a $B$-module. According to 2.1.6, the groups $\left.T_{i}(B \mid A, M)=H_{i}(L \cdot \mathscr{E}) \bigotimes_{B} M\right)$ and $\left.T^{i}(B \mid A, M)=H^{i}\left(\operatorname{Hom}_{B}(L \cdot \mathscr{E}), M\right)\right)$ are independent of the choice of $\mathscr{E}$, up to 
a canonical isomorphism $(i=0,1,2) . T_{i}(B / A, \quad)\left(\operatorname{resp} . T^{i}(B / A, \quad)\right)$ is called the $i$ th lower (resp. upper) cotangent functor of $B$ over $A$. Note that $T_{0}(B / A, M)$ $=\Omega_{B / A} \otimes_{B} M$ and $T^{0}(B / A, M)=\operatorname{Der}_{A}(B, M)$.

We list the following properties of the cotangent functors, all of which follow from the results of 2.1-2.2.

2.3.1. $M \rightarrow T_{i}(B / A, M)$ (resp. $\left.M \rightarrow T^{i}(B / A, M)\right)$ are covariant additive functors from $B$-modules to abelian groups. In particular $T_{i}(B / A, M)$ and $T^{i}(B / A, M)$ are $B$-modules.

2.3.2. Given a commutative diagram

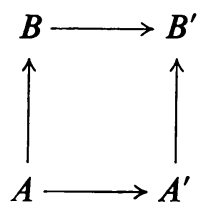

and a $B^{\prime}$-module $M^{\prime}$, there are natural homomorphisms

and

$$
T_{i}\left(B / A, M^{\prime}\right) \rightarrow T_{i}\left(B^{\prime} \mid A^{\prime}, M^{\prime}\right)
$$

$$
T^{i}\left(B^{\prime} \mid A^{\prime}, M^{\prime}\right) \rightarrow T^{\mathfrak{i}}\left(B / A, M^{\prime}\right) .
$$

Furthermore, if $B^{\prime}=B \otimes_{A} A^{\prime}$, and if either $B$ is flat over $A$ or $A^{\prime}$ is flat over $A$, then the above homomorphisms are isomorphisms.

2.3.3. If, in the diagram (*), $B^{\prime}=B \otimes_{A} A^{\prime}$, with $A^{\prime}$ flat over $A$, then for any $B$-module $M$, the induced homomorphism

$$
T_{i}(B \mid A, M) \otimes B^{\prime} \rightarrow T_{i}\left(B^{\prime} \mid A^{\prime}, M \otimes B^{\prime}\right)
$$

is an isomorphism. The homomorphisms $T^{i}(B / A, M) \otimes B^{\prime} \rightarrow T^{i}\left(B / A, M \otimes B^{\prime}\right)$ $\cong T^{i}\left(B^{\prime} \mid A^{\prime}, M \otimes B^{\prime}\right)$ (the last given by 2.3.2) induce isomorphisms

$$
T^{i}(B \mid A, M) \otimes B^{\prime} \rightarrow T^{i}\left(B^{\prime} \mid A^{\prime}, M \otimes B^{\prime}\right)
$$

provided the free extension $\mathscr{E}$ of $B$ over $A$ may be chosen so that each term of $L \cdot(\mathscr{E})$ is finitely presented over $B$. (For example, this will be the case if $A$ is noetherian and $B$ is a localization of an $A$-algebra of finite type.)

2.3.4. If $S$ is a multiplicative system in $B$, and $M$ a $B$-module, then

$$
T_{i}(B / A, M) \otimes_{B} B S^{-1} \cong T_{i}\left(B S^{-1} / A, M S^{-1}\right) .
$$

Similarly, $T^{i}\left(B S^{-1} / A, M S^{-1}\right) \cong T^{i}(B / A, M) S^{-1}$ under the finiteness conditions of 2.3.3.

2.3.5. If $A \rightarrow B \rightarrow C$ is a sequence of ring homomorphisms, and $M$ is a $C$-module, then there is an exact sequence (which is functorial in $M$ )

$$
\begin{aligned}
& T_{2}(B / A, M) \rightarrow T_{2}(C / A, M) \rightarrow T_{2}(C / B, M) \rightarrow \\
& T_{1}(B / A, M) \rightarrow T_{1}(C / A, M) \rightarrow T_{1}(C / B, M) \rightarrow \\
& T_{0}(B / A, M) \rightarrow T_{0}(C / A, M) \rightarrow T_{0}(C / B, M) \rightarrow 0 .
\end{aligned}
$$

There is a similar exact sequence for the $T^{i}$ 's, with the arrows reversed. 
2.3.6. If $0 \rightarrow M^{\prime} \rightarrow M \rightarrow M^{\prime \prime} \rightarrow 0$ is an exact sequence of $B$-modules, we get nine-term exact sequences

and

$$
\begin{aligned}
& T_{2}\left(B / A, M^{\prime}\right) \rightarrow T_{2}(B / A, M) \rightarrow T_{2}\left(B / A, M^{\prime \prime}\right) \rightarrow \\
& T_{1}\left(B / A, M^{\prime}\right) \rightarrow T_{1}(B / A, M) \rightarrow T_{1}\left(B / A, M^{\prime \prime}\right) \rightarrow \\
& T_{0}\left(B / A, M^{\prime}\right) \rightarrow T_{0}(B / A, M) \rightarrow T_{0}\left(B / A, M^{\prime \prime}\right) \rightarrow 0
\end{aligned}
$$

$$
\begin{aligned}
0 \rightarrow T^{0}\left(B / A, M^{\prime}\right) & \rightarrow T^{0}(B / A, M) \rightarrow T^{0}\left(B / A, M^{\prime \prime}\right) \rightarrow \\
T^{1}\left(B / A, M^{\prime}\right) & \rightarrow T^{1}(B / A, M) \rightarrow T^{1}\left(B / A, M^{\prime \prime}\right) \rightarrow \\
T^{2}\left(B / A, M^{\prime}\right) & \rightarrow T^{2}(B \mid A, M) \rightarrow T^{2}\left(B / A, M^{\prime \prime}\right) .
\end{aligned}
$$

2.4.1. We are now going to show that the definitions of the cotangent functors can be extended to nonaffine schemes. We will need the following lemma:

2.4.2. Lemma. Let $A$ be a ring, $B$ an A-algebra, and $C$ a B-algebra. Let $M$ be $a$ $B$-module. Assume that the induced map of Spec $C$ to $\operatorname{Spec} B$ is an open immersion. Then the natural map of $T_{i}(B / A, M) \otimes_{B} C \rightarrow T_{i}\left(C / A, M \otimes_{B} C\right)$ is an isomorphism. If we also assume that every local ring of $B$ is a localization of an A-algebra of finite type and that $A$ is noetherian, then the natural map of $T^{i}\left(C / A, M \otimes_{B} C\right)$ $\rightarrow T^{i}(B / A, M) \otimes_{B} C$ is an isomorphism.

(Ideally, the proof of this lemma should follow from the change of rings sequence and the fact that $T_{i}(C / B, M)$ and $T^{i}(C / B, M)$ are zero for all $C$-modules $M$. Unfortunately the proof (for example) that $T_{2}(B / A, M) \otimes_{B} C \rightarrow T_{2}\left(C / A, M \otimes_{B} C\right)$ is injective would require the existence of a $T_{3}(C / B, M)$ which we do not have at our disposal.)

Proof. To show that a map of $C$-modules is an isomorphism, it suffices to show that it is an isomorphism after tensoring with $C_{\mathfrak{p}}$ for every prime ideal $\mathfrak{p}$ of $C$. Let $\mathfrak{q}$ be the inverse image of $\mathfrak{p}$ in $B$. To say that $\operatorname{Spec} C \rightarrow \operatorname{Spec} B$ is an open immersion implies that the induced map of $B_{\mathfrak{q}}$ to $C_{\mathfrak{p}}$ is an isomorphism. We have now that

$$
\begin{aligned}
T_{\mathfrak{i}}(B / A, M) \otimes_{B} C \otimes_{C} C_{\mathfrak{p}} & \cong T_{\mathfrak{i}}(B / A, M) \otimes_{B} B_{\mathfrak{q}} \cong T_{\mathfrak{i}}\left(B_{\mathfrak{q}} / A, M \otimes_{B} B_{\mathfrak{q}}\right) \\
& \cong T_{i}\left(C_{\mathfrak{p}} / A, M \otimes_{B} C_{\mathfrak{p}}\right) \cong T_{i}\left(C / A, M \otimes_{B} C\right) \otimes_{C} C_{\mathfrak{p}}
\end{aligned}
$$

where the second and fourth isomorphisms are special cases of 2.3.4. The result for the $T^{i}$ follows in the same way.

2.4.3. Let $f: X \rightarrow Y$ be a morphism of preschemes. Assume that $X$ is a scheme and that $Y$ is affine, so $Y=\operatorname{Spec} A$. Let $\left\{U_{k}\right\}$ be an affine open cover of $X$. Let $U_{k}=\operatorname{Spec} B_{k}$. Since $X$ is a scheme $U_{k} \cap U_{j}$ is affine. Let $U_{k} \cap U_{j}=\operatorname{Spec} B_{k j}$. Then the map of Spec $B_{k j}$ to Spec $B_{k}$ or Spec $B_{j}$ is an open immersion. Let $F$ be a quasicoherent sheaf of $O_{x}$-modules. Let $F_{k}=F \mid U_{k}$ and let $M_{k}$ be the $B_{k}$-module corresponding to $F_{k}$. Let $F_{k j}=F \mid U_{k} \cap U_{j}$ and let $M_{k j}$ be the $B_{k j}$-module corresponding to $F_{k j}$. Let $G_{k}$ (resp. $G_{k j}$ ) be the sheaf on $U_{k}$ (resp. $U_{k j}$ ) corresponding to the 
$B_{k}$ (resp. $\left.B_{k j}\right)$ module $T_{i}\left(B_{k} \mid A, M_{k}\right)$ (resp. $\left.T_{k}\left(B_{k j} \mid A, M_{k j}\right)\right)$. We then have isomorphisms $\beta_{k j}: G_{k} \mid U_{k j} \cong G_{k j}$, by Lemma 2.4.2, and hence isomorphisms $\alpha_{k j}: G_{k} \mid U_{k j}$ $\cong G_{j} \mid U_{k j}$ by $\alpha_{k j}=\beta_{j k}^{-1} \circ \beta_{k j}$. It is clear that $\alpha_{k k}$ is the identity and $\alpha_{k j} \alpha_{j l}=\alpha_{k l}$. If we assume in addition that $Y$ is noetherian and $f$ locally of finite type, then the same situation obtains with arrows reversed and indices raised. Hence the $G_{k}$ "glue together" to form a quasi-coherent sheaf $G$ of $O_{x}$-modules.

2.4.4. Definition. $G$ is denoted by $T_{i}(X / Y, F)$. We define $T^{i}(X / Y, F)$ similarly. Note that $G$ does not depend on the open cover $\left\{U_{k}\right\}$.

2.4.5. We now wish to pass from the case where $Y$ is affine to the case where $Y$ is arbitrary. This time we will treat only the $T_{i}$, leaving the $T^{i}$ and the necessary finiteness assumptions to the reader. Let $f: X \rightarrow Y$ be an arbitrary morphism of schemes. Let $\left\{U_{k}\right\}$ be an affine open cover of $Y$. Let $U_{k}=\operatorname{Spec} B_{k}$. Since $Y$ is a scheme $U_{k} \cap U_{j}$ is affine. Let $U_{k} \cap U_{j}=\operatorname{Spec} B_{k j}$. Let $V_{k}=f^{-1}\left(U_{k}\right), V_{k j}=f^{-1}\left(U_{k j}\right)$ $=V_{k} \cap V_{j}$. Let $F$ be a quasi-coherent sheaf of $O_{x}$-modules. Let $F_{k}=F \mid V_{k}$ and $F_{k j}$ $=F \mid V_{k j}$. Let $G_{k}$ (resp. $\left.G_{k j}\right)$ be the sheaf on $V_{k}$ (resp. $\left.V_{k j}\right), T_{i}\left(V_{k} / U_{k}, F_{k}\right)$ (resp. $\left.T_{i}\left(V_{k j} / U_{k j}, F_{k j}\right)\right)$. We then have isomorphisms $\beta_{k j}: G_{k} \mid V_{k j} \cong G_{k j}$, by 2.3.3, and hence compatible isomorphisms $\alpha_{k j}: G_{k}\left|V_{k j} \cong G_{j}\right| V_{k j}$.

2.4.6. Definition. The sheaf constructed in the above fashion is defined to be $T_{i}(X / Y, F)$.

2.4.7. We have one more definition to make before we can state the proper generalization of our theorem. We content ourselves with the following: Let $g: Z \rightarrow X, f: X \rightarrow Y$ be morphisms of schemes. Let $F$ be a quasi-coherent sheaf of $O_{z}$-modules. Then it is possible to define $T_{i}(X / Y, F)$, which will be a quasi-coherent sheaf of $O_{z}$-modules, by methods analogous to those used above. (First define it for $Z, X, Y$ affine, then $X, Y$ affine, then $Y$ affine, then in general.) If we assume in addition that $Y$ is locally noetherian and $g$ and $f$ are locally of finite type, we can define quasi-coherent sheaves $T^{i}(X / Y, F)$ by the same process.

2.4.8. The above restriction that $X, Y$, and $Z$ be schemes is not essential, but greatly simplifies the construction. We also know of no serious applications of our theory to nonseparated preschemes.

We of course have the following generalizations of 2.3.4 and 2.3.6:

2.4.9. Let $Z \rightarrow Y \rightarrow X$ be a sequence of homomorphisms of schemes, and $F$ a quasi-coherent sheaf on $Z$. Then there is an exact sequence

$$
\begin{aligned}
& T_{2}(Y \mid X, F) \rightarrow T_{2}(Z \mid X, F) \rightarrow T_{2}(Z \mid Y, F) \rightarrow \\
& T_{1}(Y \mid X, F) \rightarrow T_{1}(Z \mid X, F) \rightarrow T_{1}(Z / Y, F) \rightarrow \\
& T_{0}(Y \mid X, F) \rightarrow T_{0}(Z \mid X, F) \rightarrow T_{0}(Z / Y, F) \rightarrow 0 .
\end{aligned}
$$

The sequence obtained by raising the indices and reversing the arrows is also exact, under the finiteness conditions of 2.4.7.

2.4.10. If $0 \rightarrow F_{1} \rightarrow F_{2} \rightarrow F_{3} \rightarrow 0$ is an exact sequence of quasi-coherent sheaves on $Z$, we have the nine-term exact sequences

$$
T_{2}\left(Y \mid X, F_{1}\right) \rightarrow T_{2}\left(Y \mid X, F_{2}\right) \rightarrow T_{2}\left(Y \mid X, F_{3}\right) \rightarrow T_{1}\left(Y \mid X, F_{1}\right) \rightarrow \cdots \text { etc. }
$$


and

$0 \rightarrow T^{0}\left(Y \mid X, F_{1}\right) \rightarrow T^{0}\left(Y \mid X, F_{2}\right) \rightarrow T^{0}\left(Y \mid X, F_{2}\right) \rightarrow T^{1}\left(Y \mid X, F_{1}\right) \rightarrow \cdots$ etc.

For the second sequence we assume that the usual finiteness conditions hold.

\section{Vanishing criteria.}

3.1. The vanishing of the first cotangent functor.

3.1.1. Lemma. Let $P$ be a polynomial ring over $A, M$ a $P$-module. Then $T(P / A, M)$ and $T_{i}(P \mid A, M)=0$ for $i=1$ and 2 .

Proof. This is obvious from the definition of the cotangent complex.

3.1.2. Lemma. Let $B$ be an A-algebra. Let $P$ be a polynomial ring over $A$ and $I$ an ideal in $P$ such that $B$ is isomorphic to $P / I$. Then the canonical maps (coming from the triple $(A, P, B)$ of $T_{1}(B / A, M)$ to $\operatorname{Ker}\left(I / I^{2} \otimes_{B} M \rightarrow \Omega_{P / A} \otimes_{P} M\right)$ and $\operatorname{Coker}\left(\operatorname{Hom}_{P}\left(\Omega_{P / A}, M\right) \rightarrow \operatorname{Hom}_{B}\left(I / I^{2}, M\right)\right)$ to $T^{1}(B / A, M)$ are isomorphisms.

Proof. This is an immediate consequence of Lemma 3.1.1. and the exact sequence of a triple.

3.1.3. Proposition. Let $B$ be an A-algebra. Then the following are equivalent:

(1) $T^{1}(B / A, M)=0$ for all $B$-modules $M$.

(2) $T_{1}(B \mid A, B)=0$ and $\Omega_{B / A}$ is projective.

(3) B satisfies the "lifting property" over $A$, i.e., if $C$ and $C$ ' are two $A$-algebras such that $C^{\prime} \cong C / I$ with $I^{2}=0$, and $g^{\prime}$ is an $A$-algebra homomorphism of $B$ to $C^{\prime}$, then there exists an A-algebra homomorphism $g$ from $B$ to $C$ which induces $g^{\prime}$.

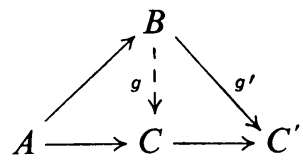

Proof. We first show (1) $\Leftrightarrow(2)$. Assume that (1) is true. From the fact that the $T^{i}$ are cohomological functors we see that the functor $T^{0}(B / A, \cdot) \cong \operatorname{Hom}_{B}\left(\Omega_{B / A}, \cdot\right)$ is an exact functor, and hence $\Omega_{B / A}$ is projective. Let $P$ be a polynomial ring over $A$ mapping onto $B$ with kernel $J$. By Lemma 3.1.2, we have the exact sequence

$$
0 \rightarrow T_{1}(B / A, B) \rightarrow J / J^{2} \rightarrow \Omega_{P / A} \otimes_{P} B \rightarrow \Omega_{B / A} \rightarrow 0 .
$$

Let $M$ be any injective $B$-module, and apply the functor $\operatorname{Hom}_{B}(\cdot, M)$ to (*). Since $T^{1}(B \mid A, M)=0$, we see that $\operatorname{Hom}_{B}\left(T_{1}(B / A, B), M\right)=0$. Choosing $M$ to contain $T_{1}(B / A, B)$, we see that $T_{1}(B / A, B)=0$.

Now assume that (2) is true. From the exact sequence $(*)$ we see that $T_{1}(B / A, B)$ $=0$ implies that $T^{1}(B / A, M)$ is isomorphic to $\operatorname{Ext}_{B}^{1}\left(\Omega_{B / A}, M\right)$ which is zero since $\Omega_{B \mid A}$ is projective.

We now prove that (1) is equivalent to (3). As above, let $B \cong P / J$. Assume that (1) is true. Let $C, C^{\prime}, I, g^{\prime}$ be given. Let $h^{\prime}$ be the induced homomorphism from $P$ 
to $C^{\prime}$. Then $h^{\prime}$ lifts to a homomorphism $h$ from $P$ to $C$. Since $I^{2}=0, I$ is a $C^{\prime}$ module and hence a $B$-module via $g^{\prime}$. Also, $h$ maps $J$ into $I$ and $J^{2}$ into zero, so it induces an element $h^{\prime \prime}$ of $\operatorname{Hom}_{B}\left(J / J^{2}, I\right)$. Since $T^{1}(B / A, I)=0, h^{\prime \prime}$ is induced by a derivation $D$ of $P$ into $I$. Since $I^{2}=0$, it is immediately checked that $h-D$ is an $A$-homomorphism of $P$ to $C$ which is zero on $J$, and hence induces a map $g$ from $B$ to $C$ lifting $g^{\prime}$.

Now assume that (3) is true. Let $x \in T^{1}(B / A, M)$. Then $x$ is induced by an element $\bar{h}$ of $\operatorname{Hom}_{B}\left(J / J^{2}, M\right) \cong \operatorname{Hom}_{P}(J, M)$. Let $K=\operatorname{Ker}(\bar{h})$. Let $\varphi: P \rightarrow P / K$ and let $C=(P / K \oplus M) /\{\varphi(z), h(z)\}$, for $z \in J$. We make $C$ into an $A$-algebra by letting $P$ act on $M$, and giving $M$ square-zero multiplication. Let $C^{\prime}=B$. Let $g^{\prime}=1_{B}$. Then we have an exact sequence of $A$-algebras

$$
0 \rightarrow M \rightarrow C \rightarrow C^{\prime} \rightarrow 0
$$

and $M^{2}=0$. Hence $g^{\prime}$ lifts to a map $g$ mapping $B$ to $C$ which induces a map $h$ of $P$ to $C$. Then $\varphi-h$ is a derivation of $P$ into $M$ which induces $h^{\prime}$, and hence $x=0$ in $T^{1}(B / A ; M)$.

3.1.4. Definition. Let $f: X \rightarrow Y$ be a morphism of preschemes. We say that $f$ is formally smooth if for every $x \in X$ there exist affine neighborhoods Spec $B$ of $x$ and Spec $A$ of $f(x)$ such that $B / A$ satisfies the equivalent conditions of Proposition 3.1.3. Note that this will then be true of any affine neighborhoods such that $B$ is an $A$-algebra.

3.1.5. THEOREM. Let $f: X \rightarrow Y$ be a morphism of finite type of locally noetherian preschemes. Then the following conditions are equivalent:

(1) $f$ is formally smooth.

(2) $T^{1}(X / Y, F)=0$ for all quasi-coherent $F$ on $X$.

(3) $T_{1}(X / Y, F)=0$ for all quasi-coherent $F$ on $X$.

(4) $f$ satisfies the "Jacobian criterion for simplicity" as stated in SGA II $\left({ }^{1}\right)$, i.e.:

Let $x \in X$, Spec $B$ an affine neighborhood of $x$, Spec $A$ an affine neighborhood of $f(x)$ such that $B$ is an A-algebra, $P$ a polynomial ring over $A$ mapping onto $B$ with kernel $J$. Then for every prime ideal $\mathfrak{p}$ of $P$ there exist generators $g_{1}, \ldots, g_{P}$ of $J_{\mathfrak{p}}$ such that $d g_{P}$ form part of a base of the free module $\Omega_{P / A} \otimes_{P} B_{\mathfrak{p}}$.

It is shown in [SGA II] that these conditions are also equivalent to

(5) $X$ is flat over $Y$ and the fibers of $f$ are absolutely nonsingular. (Recall that $a$ prescheme $V$ of finite type over a field $\mathfrak{f}$ is said to be absolutely nonsingular if all the local rings of $V \otimes_{\mathfrak{t}} \overline{\mathrm{t}}$ are regular, where $\overline{\mathrm{f}}$ denotes the algebraic closure of $\mathfrak{t}$.

(6) Locally on $X$ and $Y, f$ is the composition of an étale morphism and a morphism of the form Spec $Y\left[T_{1} \cdots T_{n}\right] \rightarrow Y$, where

$$
\text { Spec } Y\left[T_{1} \cdots T_{n}\right]=Y \times{ }_{\text {Spec } Z} \operatorname{Spec} Z \times\left[T_{1} \cdots T_{n}\right]
$$

is "affine n-space over Y."

(1) Seminaire Geometrie Algebrique (A. Grothendieck), Inst. Hautes Etudes Sci. 
Proof. All the conditions are local on $X$ and $Y$ so we may assume that $X$ $=\operatorname{Spec} B, Y=\operatorname{Spec} A$. The equivalence of (1) and (2) is contained in Proposition 3.1.3. It is easy to see, as in the proof of Proposition 3.1.3 that (3) is equivalent to:

(3') $T_{1}(B / A, B)=0$ and $\Omega_{B / A}$ is flat over $B$. If $B$ is of finite type over a noetherian $A, \Omega_{B / A}$ is of finite type over $B$ and is flat iff it is projective, so (1) $\Leftrightarrow(3)$.

It is easily seen that (4) is equivalent to the statement that the map of $J / J^{2}$ $\rightarrow \Omega_{P / A} \otimes_{P} B$ is injective, with a locally free cokernel. If we look at the exact sequence of the triple $(A, P, B)$ we see that it is equivalent to saying that $T_{1}(B / A, B)$ $=0$ and $\Omega_{B / A}$ be projective, since $\Omega_{B / A}$ is a finitely generated module over a noetherian ring. So we have shown $(1) \Leftrightarrow(4)$.

3.1.6. Definition. If $f$ satisfies the above conditions, we say $f$ is smooth, (or $X$ is smooth over $Y$ ). Note that "smooth" (French "lisse") is the new terminology for what used to be called "simple" (French "simple").

3.1.7. Proposition. (a) If $X$ is smooth over $Y$, and $Y$ is smooth over $Z$, then $X$ is smooth over $Z$.

(b) If $X$ is smooth over $Y$, and $Y^{\prime}$ is locally noetherian, then $X^{\prime}=X \times_{Y} Y^{\prime}$ is smooth over $Y^{\prime}$.

Proof. (a) follows immediately from the exact sequence of the triple $(X, Y, Z)$. To prove (b) we may assume $X=\operatorname{Spec} B, Y=\operatorname{Spec} A, Y^{\prime}=\operatorname{Spec} A^{\prime}, X^{\prime}=\operatorname{Spec} B^{\prime}$. Write $B$ as the quotient of a polynomial $\operatorname{ring} P$ over $A$ by an ideal $I$. Let $M$ be a $B^{\prime}$-module. Since $B$ is smooth over $A$, we have the exact sequence

$$
0 \rightarrow I / I^{2} \rightarrow \Omega_{P / A} \otimes_{P} B \rightarrow \Omega_{B / A} \rightarrow 0
$$

and $\Omega_{B / A}$ is projective. Let $P^{\prime}=P \otimes_{A} A^{\prime}$, and $I^{\prime}=\left(\operatorname{Ker} P^{\prime} \rightarrow B^{\prime}\right)$. Then we have a surjection from $I \otimes_{A} A^{\prime}$ to $I^{\prime}$, and hence the induced map of $I / I^{2} \otimes_{A} A^{\prime} \rightarrow I^{\prime} / I^{\prime 2}$ is a surjection. Since $\Omega_{B / A}$ is projective, we see that the map of $I / I^{2} \otimes_{B} B^{\prime} \rightarrow \Omega_{P / A}$ $\otimes_{P} B^{\prime}$ is injective. Since $I / I^{2} \otimes_{B} B^{\prime} \cong I / I^{2} \otimes_{A} A^{\prime}$ and $\Omega_{P / A} \otimes_{P} B^{\prime} \cong \Omega_{P^{\prime} / A^{\prime}} \otimes_{P^{\prime}} B^{\prime}$, we see that the map of $I^{\prime} / I^{\prime 2}$ to $\Omega_{P^{\prime} / A^{\prime}} \otimes_{P^{\prime}} B^{\prime}$ must be injective, and hence that $T_{1}\left(B^{\prime} \mid A^{\prime}, B^{\prime}\right)=0$. Since $\Omega_{B^{\prime} \mid A^{\prime}} \cong \Omega_{B / A} \bigotimes_{B} B^{\prime}$ is a projective $B^{\prime}$-module, we are done.

3.2. The vanishing of the second cotangent functor. We first consider the case when $B$ is a quotient ring of $A$. Recall that if $A$ is a local ring, a sequence of elements $a_{1}, \ldots, a_{n}$ in the maximal ideal of $A$ is said to be an $A$-sequence if $a_{1}$ is a nonzero divisor in $A$ and $a_{i}$ is a nonzero-divisor in $A /\left(a_{1} \cdots a_{i-1}\right)$ for $i>1$. If $A$ is noetherian, a necessary and sufficient condition that $\left(a_{1} \cdots a_{n}\right)$ be an $A$-sequence is that the Koszul homology $H_{1}\left(a_{1}, \ldots, a_{n}, A\right)=0$. This also shows that the property of being an $A$-sequence is independent of the order in which the $a_{i}$ are taken.

The following proposition relates $A$-sequences to the second cotangent functor:

3.2.1. Proposition. Let $A$ be a noetherian ring and $I$ an ideal of $A$. Let $B=A / I$. Then the following statements are equivalent:

(1) Let $\mathfrak{q}$ be a prime ideal in $B, \mathfrak{p}$ its inverse image in $A$. For all such $\mathfrak{q}$ and $\mathfrak{p}$, the kernel of the map $A_{\mathfrak{p}}$ to $B_{\mathfrak{q}}$ is generated by an $A_{\mathfrak{p}}$-sequence. 
(2) $T^{2}(B \mid A, M)=0$ for any $B$-module $M$.

(3) $T_{2}(B / A, B)=0$ and $I / I^{2}$ is a projective $B$-module.

(4) $T_{2}(B \mid A, M)=0$ for any $B$-module $M$.

Proof. We first show that (1) $\Rightarrow(2)$. Since the cotangent complex commutes with localization, we have $T_{2}(B / A, M)_{\mathfrak{q}} \cong T_{2}\left(B_{\mathfrak{q}} / A_{\mathfrak{p}}, M_{\mathfrak{q}}\right)$. So we may assume that $A$ and $B$ are local and $I$ is generated by an $A$-sequence, $a_{1}, \ldots, a_{n}$. Let $F=A^{n}$, and map $F$ onto $I$ in the obvious manner. Let the map be $f$, and let $U=\operatorname{Ker}(f)$. Then a cotangent complex for $B$ over $A$ can be chosen to be

$$
0 \rightarrow U / U_{0} \rightarrow F / I F \rightarrow 0 .
$$

Recall that $U_{0}$ is the image of $F \wedge F$ under the map $\varphi: x \wedge y \rightarrow f(y) x-f(x) y$. By definition of the Koszul complex with respect to $a_{1}, \ldots, a_{n}$, we see immediately that $H_{1}(a, A)$ is isomorphic to $U / U_{0}$. Hence $U / U_{0}=0$, and certainly $T^{2}(B / A, M)=0$ for any $B$-module $M$.

$(2) \Rightarrow(3)$. Since the $T^{i}$ are cohomological functors, $T^{2}(B / A, M)=0$ for all $M$ implies that the functor which takes $M$ into $T^{1}(B / A, M) \cong \operatorname{Hom}_{B}\left(I / I^{2}, M\right)$ is a right exact and hence exact functor, so $I / I^{2}$ is projective. By definition of $T_{2}(B / A, B)$ we have the exact sequence

$$
0 \rightarrow T_{2}(B / A, B) \rightarrow U / U_{0} \rightarrow F / I F \rightarrow I / I^{2} \rightarrow 0 .
$$

Let $M$ be any injective $B$-module, and apply the functor $\operatorname{Hom}_{B}(\cdot, M)$. Since $T^{2}(B / A, M)=0$, we see that $\operatorname{Hom}_{B}\left(T_{2}(B / A, B), M\right)=0$. Since any module is a submodule of an injective module, $T_{2}(B \mid A, B)=0$.

(3) $\Rightarrow$ (4). Assume (3). $T_{2}(B / A, B)=0 \Rightarrow T_{2}(B / A, M)$ is isomorphic to $\operatorname{Tor}_{1}^{B}\left(I / I^{2}, M\right)$, which is zero since $I / I^{2}$ is projective, hence flat. Assume (4). $T_{2}(B / A, B)=0$ $\Rightarrow \operatorname{Tor}_{1}^{B}\left(I / I^{2}\right) \cong T_{2}(B / A, M)=0$ for all $M$, which implies $I / I^{2}$ is flat.

(3) $\Rightarrow(1)$. Since the cotangent functors and flatness localize, we may assume that $A$ and $B$ are local, $\mathfrak{q}=$ the maximal ideal in $B$ and $\mathfrak{p}=$ the maximal ideal in $A$. Since $B$ is local, $I / I^{2}$ is a free $B$-module. Let $a_{1}, \ldots, a_{n}$ be elements of $I$ whose residue classes form a basis for $I / I^{2}$. By Nakayama's Lemma, the $a_{i}$ generate $I$. Let $F \wedge F \rightarrow^{\Phi} F \rightarrow^{F} A$ be the Koszul complex with respect to $\left(a_{1}, \ldots, a_{n}\right)$. Let $U=\operatorname{Ker} f$ and $U_{0}=\operatorname{Im} \varphi$. Then we may choose the cotangent complex of $B$ over $A$ to be

$$
0 \rightarrow U / U_{0} \rightarrow F / I F \rightarrow 0,
$$

and we have the exact sequence

$$
0 \rightarrow T_{2}(B / A, B) \rightarrow U / U_{0} \rightarrow F / I F \rightarrow I / I^{2} \rightarrow 0 .
$$

Since $F$ is a free module on the generators of $I$, and $I / I^{2}$ is a free module on $\bar{a}_{1}, \ldots, \bar{a}_{n}$, the map of $F / I F$ to $I / I^{2}$ is an isomorphism. Hence $H_{1}(a, A)=U / U_{0}$ is isomorphic to $T_{2}(B / A, B)=0$ by assumption. Hence the $a_{i}$ form an $A$-sequence.

3.2.2. Corollary. Let $A$ be a noetherian ring and $B$ an A-algebra of finite type. Then the following conditions are equivalent: 
(1) There exists a polynomial ring $P$ over $A$ and an ideal $I$ in $P$ such that $B$ is isomorphic to $P / I$ and the pair $(P, B)$ satisfies the conditions of the preceding proposition.

(2) If $Q$ is any polynomial ring over $A$ and $J$ is an ideal in $Q$ such that $B$ is isomorphic to $Q / J$, then the pair $(Q, B)$ satisfies the conditions of the preceding proposition.

(3) $T^{2}(B \mid A, M)=0$ for any $B$-module $M$.

(4) $T_{2}(B \mid A, B)=0$ and $I / I^{2}$ is locally free, where $I$ is the kernel of a map onto $B$ of any (or, equivalently, some) polynomial ring over $A$.

(5) $T_{2}(B / A, M)=0$ for all $B$-modules $M$.

Proof. For any polynomial ring $P$ over $A$ which has a quotient isomorphic to $B$, it immediately follows from the exact sequence of the triple $(A, P, B)$ that the natural map of $T_{2}(B / A, M)$ to $T_{2}(B / P, M)$ (resp. $T^{2}(B / P, M)$ to $\left.T^{2}(B / A, M)\right)$ is an isomorphism for any $B$-module $M$. The result then follows directly from the preceding proposition.

3.2.3. Definition. If the above conditions are satisfied, we say $B$ is Koszul over $A$.

3.2.4. Definition. Let $f: X \rightarrow Y$ be a morphism of finite type of locally noetherian preschemes. We say that $f$ is Koszul if for every $x \in X$ there exist affine open neighborhoods Spec $B$ of $x$ and Spec $A$ of $f(x)$ such that $B$ is an $A$-algebra and $B$ is Koszul over $A$.

It follows immediately from the two preceding propositions that if $f$ is Koszul, then for any choice of affine open neighborhoods Spec $B$ of $x$ and Spec $A$ of $f(x)$ such that $B$ is an $A$-algebra, $B$ is Koszul over $A$. Also, " $f$ is Koszul," " $T_{2}(X / Y, F)$ $=0$ for all quasi-coherent sheaves $F$ " and " $T^{2}(X / Y, F)=0$ for all quasi-coherent sheaves $F$ "' are all equivalent.

3.2.5. Proposition. (a) If $X$ is Koszul over $Y$, and $Y$ is Koszul over $Z$, then $X$ is Koszul over $Z$.

(b) If $X$ is Koszul over $Y$, and $Y^{\prime}$ is locally noetherian and flat over $Y$, then $X^{\prime}=X \times_{Y} Y^{\prime}$ is Koszul over $Y^{\prime}$.

Proof. (a) follows immediately from the exact sequence of the triple $(X, Y, Z)$. (b) is a consequence of the fact that the cotangent complex commutes with flat base extension. It is easily seen that (b) is false if the assumption of flatness is dropped.

3.3. The local case. When $X=\operatorname{Spec} B$ is the spectrum of a noetherian local ring $B$, the vanishing criteria can be sharpened, as follows:

3.3.1. Proposition. Let $A$ be a noetherian ring and $B$ a noetherian local ring which is the localization of an algebra $C$ of finite type over $A$ at a prime ideal $\mathfrak{p}$. Let $K$ be the residue field of $B$. Then the following conditions are equivalent:

(1) $C$ is smooth over $A$ in a neighborhood of $\mathfrak{p}$.

(2) $T^{1}(B / A, M)=0$ for all $B$-modules $M$.

(3) $T^{1}(B / A, K)=0$.

(4) $T_{1}(B / A, K)=0$. 
Proof. (1) $\Leftrightarrow(2)$ follows immediately from the definition of "smooth" and from the fact that the cotangent functors commute with localization, as does (1) $\Rightarrow$ (4). $(2) \Rightarrow(3)$ is obvious. We now show (3) $\Rightarrow(1)$. Write $K$ as a submodule of an injective module $I$, so there is an exact sequence $0 \rightarrow K \rightarrow I \rightarrow Q \rightarrow 0$. Since $T^{1}(B / A, K)=0$, we have the exact sequence

$$
0 \longrightarrow \operatorname{Hom}_{B}\left(\Omega_{B / A}, K\right) \longrightarrow \operatorname{Hom}_{B}\left(\Omega_{B / A}, I\right) \stackrel{\varphi}{\longrightarrow} \operatorname{Hom}_{B}\left(\Omega_{B / A}, Q\right) \longrightarrow 0 .
$$

However the cokernel of $\varphi$ is by definition $\operatorname{Ext}_{B}^{1}\left(\Omega_{B / A}, K\right)$, which must therefore $=0$. Exactly as in the case of Tor $_{1}$, it follows that $\Omega_{B / A}$ is a free $B$-module. (It is clear that $\Omega_{B / A}$ is a finitely generated $B$-module.) We will now show that $T_{1}(B / A, B)=0$. We write $B$ as the quotient of a polynomial ring $P$ over $A$ by an ideal $J$. Then

$$
T^{1}(B / A, K) \cong \operatorname{Coker}\left(\operatorname{Hom}_{B}\left(\Omega_{P / A} \otimes_{P} B, K\right) \rightarrow \operatorname{Hom}_{B}\left(J / J^{2}, K\right)\right) .
$$

From the exact sequence

$$
0 \rightarrow T_{1}(B / A, B) \rightarrow J / J^{2} \rightarrow \Omega_{P / A} \otimes_{P} B \rightarrow \Omega_{B / A} \rightarrow 0,
$$

using the fact that $\Omega_{P / A} \otimes_{P} B$ and $\Omega_{B / A}$ are projective, we find that the above cokernel is isomorphic to $\operatorname{Hom}_{B}\left(T_{1}(B \mid A, B), K\right)$. Hence $\operatorname{Hom}_{B}\left(T_{1}(B / A, B), K\right)=0$, and since $T_{1}(B / A, B)=T_{1}(C / A, C)_{\mathfrak{p}}$ is a finitely generated $B$-module, this implies that $T_{1}(B \mid A, B)=0$. Since $\Omega_{B / A}=\left(\Omega_{C / A}\right.$ localized at $\left.\mathfrak{p}\right)$ is free, $\Omega_{C / A}$ is free in a neighborhood of $\mathfrak{p}$ and $T_{1}(C / A, C)$ is zero in a neighborhood of $\mathfrak{p}$. Hence $C$ is smooth over $A$ in a neighborhood of $\mathfrak{p}$.

We now show that $(4) \Rightarrow(1)$. Since the pair $\left(T_{1}, T_{0}\right)$ is part of a connected sequence of homological functors, and $T_{0} \cong \Omega_{B / A} \otimes_{B} \cdot$, we see that $T_{1}(B / A, K)$ maps onto $\operatorname{Tor}_{1}^{B}\left(\Omega_{B / A}, K\right)$. Since $T_{1}(B \mid A, K)$ is zero, so is $\operatorname{Tor}_{1}^{B}\left(\Omega_{B / A}, K\right)$ and hence $\Omega_{B / A}$ is free. But then the functor $T_{1}(B / A, \cdot)$ is a covariant right exact functor, and hence isomorphic to $T_{1}(B \mid A, B) \otimes \cdot$. Hence $T_{1}(B / A, B) \otimes_{B} K=0$, and by Nakayama's Lemma, $T_{1}(B \mid A, B)=0$. Hence, as above, $C$ is smooth over $A$ in a neighborhood of $\mathfrak{p}$.

We now give the exactly analogous proposition for the second cotangent functor:

3.3.2. Proposition. Let $A$ be a noetherian ring and $B$ a noetherian local ring which is the localization of an algebra $C$ of finite type over $A$ at a prime ideal $\mathfrak{p}$. Let $K$ be the residue field of $B$. Then the following conditions are equivalent:

(1) $C$ is Koszul over $A$ in a neighborhood of $\mathfrak{p}$.

(2) $T^{2}(B / A, M)=0$ for all $B$-modules $M$.

(3) $T^{2}(B / A, K)=0$.

(4) $T_{2}(B / A, K)=0$.

Proof. The proof is identical to the preceding one, after observing that, replacing $A$ by a polynomial ring over $A$ and localizing if necessary, we may assume that $B$ is a quotient of $A$. We write $B=A / I$, we write $I$ as the quotient of a free $B$-module $I=F / U$, we replace $\Omega_{B / A}$ by $I / I^{2}, \Omega_{P / A} \otimes_{P} B$ by $F / I F, I / I^{2}$ by $U / U_{0}$, and argue as above. 
As a corollary of the above results in the local case, we can strengthen our earlier theorems in the global case. We have in fact:

3.3.3. THEOREM. Let $X$ and $Y$ be locally noetherian preschemes, $X$ of finite type over $Y$. Then $X$ is smooth over $Y$ iff $T_{1}(X / Y, K(A))=0$ for all local rings $A$ of $X$. $X$ is Koszul over $Y$ iff $T_{2}(X / Y, K(A))=0$ for all local rings $A$ of $X$.

The proof is immediate.

As another corollary of the results of this section, we have the following theorem:

3.3.4. Theorem. Let $A, B, C$ be three noetherian local rings, $I$ and $J$ ideals in $A$, $K$ an ideal in $B$ such that $B=A / I, C=A / J \cong B / K$, i.e., $K=J / I$. Assume that $J$ is generated by an $A$-sequence, and $K$ is generated by a $B$-sequence. Then $I$ is generated by an A-sequence.

Proof. We look at the exact sequence of the triple $(A, B, C)$ applied to the $C$-module $C$. We have

$$
T_{2}(C / B, C) \rightarrow I / I^{2} \otimes_{B} C \rightarrow J / J^{2} \rightarrow K / K^{2} \rightarrow 0 .
$$

The hypotheses imply that $T_{2}(C / B, C)=0$ and that $J / J^{2}$ and $K / K^{2}$ are free $C$-modules. Also, we know that $\operatorname{rank}\left(J / J^{2}\right)=\operatorname{dim} A-\operatorname{dim} C$ and $\operatorname{rank}\left(K / K^{2}\right)$ $=\operatorname{dim} B-\operatorname{dim} C$. It follows that $I / J I \cong I / I^{2} \otimes_{B} C$ is free of rank equal to $\operatorname{dim} A$ $-\operatorname{dim} B$. Hence by Nakayama's Lemma, $I$ is generated by $(\operatorname{dim} A-\operatorname{dim} B)$ generators.

Let $\bar{y}_{1}, \ldots, \bar{y}_{r}$ be a $B$-sequence generating $K$ and let $y_{1}, \ldots, y_{r}$ be elements of $A$ mapping onto $\bar{y}_{1}, \ldots, \bar{y}_{r}$. Let $x_{1}, \ldots, x_{s}$ be a minimal set of generators for $I$. Then $\left(x_{1} \cdots x_{s}, y_{1} \cdots y_{r}\right)=J$ and $s+r=\operatorname{dim} A-\operatorname{dim} C$. So the residue classes of the $x_{i}$ 's and the $y_{i}$ 's form a basis for $J / J^{2}$, and hence form an $A$-sequence. Since any subset of an $A$-sequence is an $A$-sequence, we are done.

3.4. The cotangent functors and field extensions. We will show now that the second cotangent functor vanishes for any field extension, and that the first cotangent functor vanishes iff the extension is separable. Precisely, we have the following

3.4.1. TheOREM. Let $K$ be a field and $L$ an overfield. Then $T^{2}(L / K, M)$ $=T_{2}(L / K, M)=0$ for all $L$-modules $M$, and the following conditions are equivalent:

(a) $L$ is separable over $K$.

(b) $T^{1}(L / K, M)=0$ for all $L$-modules $M$.

(b') $T^{1}(L / K, L)=0$.

(c) $T_{1}(L / K, M)=0$ for all L-modules $M$.

(c') $T_{1}(L / K, L)=0$.

Proof. To prove the first statement, we reduce first to the case when $L$ is finitely generated over $K$, since the cotangent complex commutes with direct limits. By using the exact sequence of a triple, we may assume $L=K(x)$. If $x$ is transcendental over $K$, then $L$ is the localization of a polynomial ring over $K$, and $T_{2}$ and $T^{2}$ vanish by 3.1.1 and 2.3.4. 
To prove the equivalence of the five conditions, first observe that $(b) \equiv\left(b^{\prime}\right)$ and $(c) \equiv\left(c^{\prime}\right)$ are trivial. We will show that $(a) \equiv(c)$ and leave the analogous proof that (a) $\equiv(b)$ to the reader. So we first assume (a). Since the cotangent complex commutes with direct limits, we may assume $L$ is finitely separably generated over $K$. (We know $L$ is separable over $K$ iff every finitely generated subextension of $L$ over $K$ is separably generated.) By the exact sequence of a triple, we reduce to the case $L=K\left(X_{1}, \ldots, X_{n}\right), X_{i}$ independent transcendentals, and the case when $L$ is a finite separable extension of $K$. In the first case, $L$ is the localization of a polynomial algebra over $K$, and hence $T_{1}(L / K, M)=0$ for any $M$. In the second case, we write $L=K[x]$, where $f(x)=0, f^{\prime}(x) \neq 0$. Let $P=K[X]$. Then we have the exact sequence

$$
0 \longrightarrow T_{1}(L / K, L) \longrightarrow(f) /\left(f^{2}\right) \stackrel{d}{\longrightarrow} \Omega_{P / K} \otimes_{P} L \longrightarrow \Omega_{L / K} \longrightarrow 0 .
$$

Since $(f) /\left(f^{2}\right)$ and $\Omega_{P / K} \otimes_{P} L$ are free $L$-modules of rank one, and $d f=f^{\prime}(x) d x \neq 0$, $T_{1}(L / K, L)=0$, and hence $T_{1}(L / K, M)=0$ for any $M$.

We now assume (c). We want to prove that any finitely generated subextension $L^{\prime}$ of $L / K$ is separably generated. Using the first part of the theorem, together with the exact sequence of the triple $\left(K, L^{\prime}, L\right)$ we see that $T_{1}\left(L^{\prime} / K, L\right)=0$ and hence $T_{1}\left(L^{\prime} \mid K, M\right)=0$ for all $L^{\prime}$-modules $M$, i.e., we may assume that $L$ is finitely generated over $K$. By induction on the number of generators, using the exact sequence of a triple repeatedly, the following statement is easily proved:

$$
\operatorname{tr} \operatorname{deg} L / K=\operatorname{dim} \Omega_{L / K}-\operatorname{dim} T_{1}(L / K, L) .
$$

Hence $T_{1}(L / K, L)=0 \Rightarrow \Omega_{L / K}$ is generated by $d x_{1}, \ldots, d x_{n}$ where $n=\operatorname{tr} \operatorname{deg}(L / K)$. Let $K^{\prime}=K\left(x_{1}, \ldots, x_{n}\right)$. Then the exact sequence of the triple $\left(K, K^{\prime}, L\right)$ shows that $\Omega_{L / K^{\prime}}=0$, which implies that $T_{1}\left(L / K^{\prime}, L\right)=0$ and $L$ is a finite algebraic extension of $K^{\prime}$. If $L$ were not separable over $K^{\prime}$, there would be an element $\alpha \in L$ such that $\alpha^{p} \in K^{\prime}, \alpha \notin K^{\prime},\left(p=\operatorname{ch} K^{\prime}\right)$. Then direct computation shows that $T_{1}\left(K^{\prime}(\alpha) / K^{\prime}\right.$, $\left.K^{\prime}(\alpha)\right) \neq 0$, but from the exact sequence of the triple $\left(K^{\prime}, K^{\prime}(\alpha), L\right)$ it follows that $T_{1}\left(K^{\prime}(\alpha) / K^{\prime}, K^{\prime}(\alpha)\right)=0$. Hence $L$ is separable over $K^{\prime}$, and $L$ is separably generated over $K$, since $K^{\prime}$ is clearly a purely transcendental extension of $K$.

3.5. Simplicity over a field. We are now in a position to show that, over a field, "smooth" is equivalent to "absolutely nonsingular." More precisely, we have:

3.5.1. TheOREM. Let $K$ be a field, $K^{*}$ the algebraic closure of $K$, and $X$ a prescheme of finite type over $K$. Then the following conditions are equivalent:

(1) $X$ is smooth over $K$.

(2) $X \otimes_{K} K^{*}$ is a regular prescheme.

Proof. $(1) \Rightarrow(2)$. Since smoothness is preserved under base extensions, we may assume that $K^{*}=K$. Let $A$ be a local ring of $X$. Let $K^{\prime}$ be the residue field of $A$. Consider the exact sequence of the triple $\left(K, A, K^{\prime}\right)$ applied to the $K^{\prime}$-module $K^{\prime}$ :

$$
T_{2}\left(K^{\prime} \mid K, K^{\prime}\right) \rightarrow T_{2}\left(K^{\prime} \mid A, K^{\prime}\right) \rightarrow T_{1}\left(A \mid K, K^{\prime}\right) .
$$


Since the cotangent functors localize, $T_{1}\left(A \mid K, K^{\prime}\right)=0$, and by the previous theorem, $T_{2}\left(K^{\prime} \mid K, K^{\prime}\right)=0$, so $T_{2}\left(K^{\prime} \mid A, K^{\prime}\right)=0$, i.e., $K^{\prime}$ is a complete intersection in $A$, i.e., $A$ is regular.

(2) $\Rightarrow(1)$. Let $X^{*}=X \otimes_{K} K^{*}$. We first show that $X^{*}$ is smooth over $K^{*}$. It suffices to show that for every local ring $B$ of $X^{*}, T_{1}\left(B / K^{*}, K(B)\right)=0$, where $K(B)$ denotes the residue field of $B$. By the exact sequence of the triple $\left(K^{*}, B, K(B)\right)$ we know that

$$
T_{2}(K(B) / B, K(B)) \rightarrow T_{1}\left(B / K^{*}, K(B)\right) \rightarrow T_{1}\left(K(B) / K^{*}, K(B)\right)
$$

is exact. Since $B$ is regular, the first term is zero. Since $K^{*}$ is algebraically closed, $K(B)$ is separable over $K^{*}$, and the last term is zero. Hence the middle term is zero, i.e., $X^{*}$ is smooth over $K^{*}$. But, since the cotangent complex commutes with flat base extension,

$$
T_{1}(X \mid K, F) \otimes_{K} K^{*} \cong T_{1}\left(X^{*} / K^{*}, F \otimes_{X} X^{*}\right)=0,
$$

so $T_{1}(X \mid K, F)=0$, i.e., $X$ is smooth over $K$.

4. Extensions and deformations. As is usual in cohomology theories, the cotangent modules $T^{1}$ and $T^{2}$ may be viewed as modules of "infinitesimal extensions" of length one and two respectively (4.1 and 4.2), and $T^{2}$ is the module of obstructions for the existence of noninfinitesimal one term extensions. These facts are reinterpreted more geometrically in terms of "infinitesimal deformations" in 4.3.

4.0. Notation. If $x$ is an object of some set on which there is defined an equivalence relation, we denote by $\operatorname{cl}(x)$ the equivalence class of $x$, when the context is clear. If $p_{1}: E_{1} \rightarrow E$ and $p_{2}: E_{2} \rightarrow E$ are ring homomorphisms, we denote by $E_{1} \times_{E} E_{2}$ the ring of all pairs $\left(e_{1}, e_{2}\right)\left(e_{i} \in E_{i}\right)$ such that $p_{1}\left(e_{1}\right)=p_{2}\left(e_{2}\right)$, with pairwise addition and multiplication.

\subsection{Two term extensions.}

4.1.0 Definition. Let $A$ be a ring, $B$ an $A$-algebra and $M$ a $B$-module. By a two term (infinitesimal) extension of $B$ over $A$ by $M$, we mean an extension in the sense of 2.1:

$$
\mathscr{E}: 0 \longrightarrow M \stackrel{i}{\longrightarrow} J \stackrel{j}{\longrightarrow} E \stackrel{k}{\longrightarrow} B \longrightarrow 0
$$

in which $E_{2}=M$, viewed as an $E$-module via $k$. Two such extensions, $\mathscr{E}$ and $\mathscr{E}^{\prime}$ are equivalent if there exist extensions $\mathscr{E}_{0}=\mathscr{E}, \mathscr{E}_{1}, \ldots, \mathscr{E}_{n}=\mathscr{E}^{\prime}$ of $B$ over $A$ by $M$ and homomorphisms

$$
\mathscr{E}_{0} \rightarrow \mathscr{E}_{1} \leftarrow \mathscr{E}_{2} \rightarrow \cdots \leftarrow \mathscr{E}_{n-1} \rightarrow \mathscr{E}_{n}
$$

all reducing to the identity on $M$ and $B$. In other words, equivalence among two term extensions is the smallest equivalence relation containing all pairs $\left(\mathscr{E}, \mathscr{E}^{\prime}\right)$ which are connected by a homomorphism (either way) reducing to the identity on both ends. The extension $0 \rightarrow M \rightarrow^{\text {id }} M \rightarrow^{0} B \rightarrow^{\text {id }} B \rightarrow 0$ is called trivial. 
4.1.1. Definition. Let $\operatorname{Ex}^{2}(B / A, M)$ denote the set of equivalence classes of two term extensions.

Given an extension $\mathscr{E}$ as above, there is a natural homomorphism $k^{*}$ from the cohomology module $H^{i}\left(\operatorname{Hom}\left(L^{\cdot}(\mathscr{E}), M\right)\right)$ to $T^{i}(B / A, M)$ (by 2.1.7). Let $\varepsilon$ be the class of the identity endomorphism of $M$ in $H^{2}\left(\operatorname{Hom}\left(L^{\cdot}(\mathscr{E}), M\right)\right.$ ) (=Coker $\left.\operatorname{Hom}_{E}(J, M) \rightarrow \operatorname{Hom}_{B}(M, M)\right)$. Then

\subsubsection{THEOREM. The assignment $\mathscr{E} \rightarrow k^{*}(\varepsilon)$ induces a bijection}

$$
\rho: \operatorname{Ex}^{2}(B / A, M) \rightarrow T^{2}(B / A, M)
$$

in which the class of the trivial extension corresponds to 0.

Proof. Let

$$
\mathscr{F}: \quad 0 \longrightarrow \mathrm{T}_{2} \stackrel{\bar{i}}{\longrightarrow} F_{1} \stackrel{\bar{j}}{\longrightarrow} P \stackrel{\bar{k}}{\longrightarrow} B \longrightarrow 0
$$

be a fixed free extension of $B$ over $A$ (2.13). Given $\mathscr{E}$ we get a homomorphism $\alpha$ : $\mathscr{F} \rightarrow \mathscr{E}$ unique up to homotopy, and the class of $\alpha_{2}$ in $T^{2}(B / A, M)$ is $k^{*}(\varepsilon)$. Thus if $\beta: \mathscr{E} \rightarrow \mathscr{E}^{\prime}$ is a homomorphism (reducing to the identity on both ends), we may choose $\alpha^{\prime}: \mathscr{F} \rightarrow \mathscr{E}^{\prime}$ to be $\alpha^{\prime}=\beta \circ \alpha$, so that $\alpha_{2}=\alpha_{2}^{\prime}, k^{*}(\varepsilon)=k^{\prime *}\left(\varepsilon^{\prime}\right)$; therefore $k^{*}(\varepsilon)$ depends only on the equivalence class of $\mathscr{E}$ and $\rho$ is well defined. Clearly $\rho$ (class of trivial extension) $=0$.

On the other hand, given $e \in T^{2}(B / A, M)$, choose $\alpha_{2}: F_{2} \rightarrow M$ inducing $e$. Put $J=\left(F_{1} \oplus M\right) / K$ where $K$ is the submodule of all pairs $\left(i x_{2},-\alpha_{2} x_{2}\right)$, for $x_{2} \in F_{2}$, and let $E=P, i(m)=$ class of $(0, m), j$ (class of $\left.x_{1}, m\right)=j\left(x_{1}\right) \in E=P, k=k$. Then it is easy to see that these data define an extension $\mathscr{E}$ of $B$ over $A$ by $M$, and that there is a homomorphism $\alpha: \mathscr{F} \rightarrow \mathscr{E}$ whose second component is $\alpha_{2}$. Thus $e=$ class $\alpha_{2}=k^{*}(\varepsilon)$.

It remains to show that the equivalence class of $\mathscr{E}$ depends only on the class of $e$ in $T^{2}(B \mid A, M)$. If $\alpha_{1}: F_{1} \rightarrow M$ is a homomorphism, and $\alpha_{2}^{\prime}=\alpha_{2}+\alpha_{1} \circ \bar{j}$, let $\mathscr{E}^{\prime}$ be the extension constructed from $\alpha_{2}^{\prime}$ as above. Then the endomorphism $\left(x_{1}, m\right)$ $\rightarrow\left(x_{1},-\alpha_{1}\left(x_{1}\right)+m\right)$ of $F_{1} \oplus M$ induces $J \rightarrow J^{\prime}$, which in turn induces a homomorphism $\mathscr{E} \rightarrow \mathscr{E}^{\prime}$ reducing to the identity on both ends. Thus $\mathscr{E}$ and $\mathscr{E}^{\prime}$ are equivalent, so that $e \rightarrow \mathscr{E}$ is a well-defined map, inverse to $\rho$. Q.E.D.

4.2. One term extensions.

4.2.0. Definition. Let $A$ be a ring, $B$ an $A$-algebra, and $M$ a $B$-module. By a one term or short extension of $B$ over $A$ by $M$ we mean an exact sequence:

$$
0 \longrightarrow M \stackrel{i}{\longrightarrow} E \stackrel{k}{\longrightarrow} B \longrightarrow 0
$$

where $E$ is an $A$-algebra (commutative with identity) $k$ is a surjection of $A$-algebras, and $M$ is regarded as a square zero ideal in $E$.

Thus a short extension is a degenerate two term extension, obtained by putting $E_{2}=0, E_{1}=M$ and requiring that $e_{1}(x) y=0\left(x, y \in E_{1}\right)$ in 2.1.4.

If $E^{\prime}$ is another short extension of $B$ over $A$ by $M$ we say that $E$ and $E^{\prime}$ are 
equivalent if there exists a homomorphism $\theta: E \rightarrow E^{\prime}$ of $A$ algebras inducing a commutative diagram

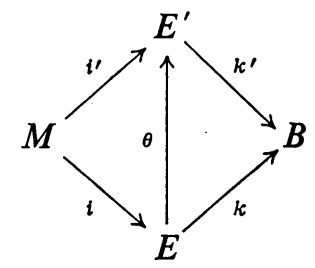

( $\theta$ must then be an isomorphism.)

4.2.1. Definition. Let $\operatorname{Ex}^{1}(B / A, M)$ be the set of equivalence classes of short extensions of $B$ over $A$ by $M$.

Given an extension $E$, the homomorphism $h: A \rightarrow E$ induces

$$
h^{*}: \operatorname{Hom}_{B}(M, M)=T^{1}(B / E, M) \rightarrow T^{1}(B / A, M) .
$$

Let id be the identity endomorphism of $M$. Then

4.2.2. TheOREM. The assignment $E \rightarrow h^{*}(\mathrm{id})$ induces a bijection

$$
\rho: \operatorname{Ex}^{1}(B / A, M) \cong T^{1}(B / A, M)
$$

in which the class of the trivial extension $0 \rightarrow M \rightarrow M \oplus B \rightarrow B \rightarrow 0$ corresponds to 0 .

The proof is quite similar to that of 4.1.2, and we omit it.

4.2.3. Noninfinitesimal short extensions. Let $J$ be a commutative $A$-algebra, not necessarily having an identity. By a multiplication of $J$ we mean an $A$-linear map $\sigma: J \rightarrow J$ such that $\sigma(x y)=\sigma(x) \cdot y$ for $x$ and $y$ in $J$. Define the product of two multiplications by composition, and let $M_{J}$ be a set of all multiplications $\sigma$ such that $\sigma \tau=\tau \sigma$ for every multiplication $\tau$. Notice that $M_{J}$ is a commutative $A$-algebra, with unit. Then we get a two term extension

$$
\mathscr{E}_{\mathrm{J}}: 0 \longrightarrow C_{\mathrm{J}} \stackrel{i}{\longrightarrow} \mathrm{J} \stackrel{j}{\longrightarrow} M_{\mathrm{J}} \stackrel{k}{\longrightarrow} E_{\mathrm{J}} \longrightarrow 0
$$

where $C_{J}=$ the set of $x \in J$ such that $x y=0$ for all $y$ in $J, j(x)=\sigma_{x}: y \rightarrow x y . j(J)$ is by definition the algebra of interior multiplications, and $E_{J}$ the algebra of exterior multiplications. Observe also that $C_{J}$ is an $E_{J}$-module.

4.2.4. Definition. If $J$ is a commutative $A$-algebra as above, and $B$ an $A$-algebra (commutative with identity) then an extension of $B$ over $A$ by $J$ is an exact sequence

$$
0 \longrightarrow J \stackrel{i}{\longrightarrow} E \stackrel{j}{\longrightarrow} B \longrightarrow 0
$$

where $E$ is a commutative $A$-algebra with identity, $j(1)=1$ and $i(s t)=i(s) i(t)$ for $\rho, t \in J$. Such an extension $E$ defines a homomorphism $\mu: B \rightarrow E_{J}$ induced from the action of $E$ on $J$.

Now given $J$ and $\mu: B \rightarrow E_{J}$, let $\operatorname{Ex}(\mu, J)$ denote the (possibly empty) set of 
isomorphism classes of extensions with given $\mu$. Notice that $C_{J}$ becomes a $B$ module via $\mu$, and that $\mu$ induces a homomorphism

$$
\mu^{*}: \quad T^{2}\left(E_{J} / A, C_{J}\right) \rightarrow T^{2}\left(B / A, C_{J}\right) .
$$

4.2.5. Let $A$ be a ring, $B$ and $J$ commutative $A$-algebras ( $B$ with (1) and $\mu$ : $B \rightarrow E_{J}$ a homomorphism of $A$-algebras $(\mu(1)=1)$. Then

(i) $\operatorname{Ex}(\mu, J) \neq \varnothing$ if and only if the element $\mu^{*}\left(\operatorname{cl}\left(\mathscr{E}_{J}\right)\right)$ in $T^{2}\left(B / A, C_{J}\right)$ is zero.

(ii) If $\mu^{*}\left(\operatorname{cl}\left(\mathscr{E}_{J}\right)\right)=0$, then there is a group action $\theta: T^{1}\left(B / A, C_{J}\right) \times \operatorname{Ex}(\mu, J)$ $\rightarrow \operatorname{Ex}(\mu, J)$ which makes Ex a principal homogeneous space under $T^{1}$ (i.e., for fixed $e^{\prime} \in \mathrm{Ex}, e \rightarrow \theta\left(e, e^{\prime}\right)$ induces a bijection $\left.T^{1} \cong \mathrm{Ex}\right)$.

Before beginning the proof we need the following observations:

4.2.6. Let $\mu: B \rightarrow C$ be a homomorphism of $A$-algebras, $M$ a $C$-module and $e \in T^{2}(C / A, M)$. If $\mathscr{E}$ is an extension corresponding to $e$, then, as in the theory of extensions in an abelian category [11, p. 63], the extension $\mathscr{G}$ corresponding to $\mu^{*}(e)$ is characterized up to equivalence by the property that there exists a $\beta$ : $\mathscr{G} \rightarrow \mathscr{E}$ extending $\mu$ such that $\beta_{2}=$ id on $M$. Hence if $\mathscr{F}: 0 \rightarrow F_{2} \rightarrow F_{1} \rightarrow P \rightarrow B \rightarrow 0$ is a free extension of $B$ over $A$, and $\alpha: \mathscr{F} \rightarrow \mathscr{E}$ extends $\mu$, it is easy to see that $\mu^{*}(e)$ is the residue class of $\alpha_{2}$ in $T^{2}(B / A, M)=$ Coker: $\operatorname{Hom}_{P}\left(F_{1}, M\right)$ $\rightarrow \operatorname{Hom}_{P}\left(F_{2}, M\right)$ (see diagram below).

Proof of 4.2.5. (i) Let $\mathscr{F}$ be a free extension of $B$ over $A$, and let $\alpha: \mathscr{F} \rightarrow \mathscr{E}_{J}$ extend $\mu: B \rightarrow \mathscr{E}_{\mathrm{J}}$.

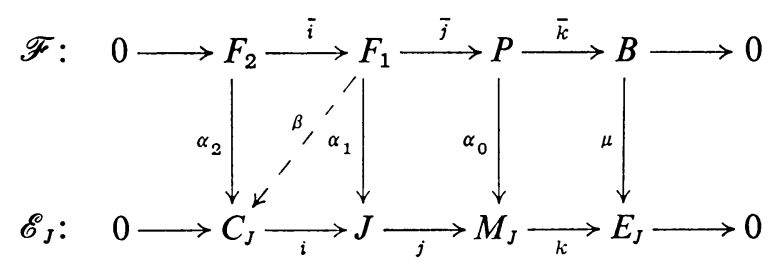

Thus by 4.2 .6 above, $\mu^{*}\left(\operatorname{cl}\left(\mathscr{E}_{J}\right)\right)=0$ if and only if there exists a $\beta: F_{1} \rightarrow C_{J}$ such that $\beta \bar{l}=\alpha_{2}$. If such a $\beta$ exists, put $E=(P \oplus J) / K$ where $K$ is the set of pairs of the form $\left(j x_{1},-\alpha_{1} x_{1}+i \beta x_{1}\right)$ with $x_{1} \in F_{1}$, and where multiplication in $P \oplus J$ is defined by $(p, t)(q, s)=\left(p q, t s+\alpha_{0}(p) s+\alpha_{0}(q) t\right)$. Routine checking then shows that we have defined an extension $0 \rightarrow J \rightarrow E \rightarrow B \rightarrow 0$ of $B$ by $J$.

Conversely, if $0 \rightarrow J \rightarrow E \rightarrow B \rightarrow 0$ is an extension, we can regard it as a degenerate two term extension, $\mathscr{E}$, with last term (0); then we have homomorphisms $\gamma: \mathscr{F} \rightarrow \mathscr{E}$ and $\delta: \mathscr{E} \rightarrow \mathscr{E}_{J}$ (where $\delta$ extends $\mu$ ). Since we may assume that $\alpha=\delta \gamma$, and since $\operatorname{cl}(\mathscr{E})=0$, it follows that $\mu^{*}\left(\operatorname{cl}\left(\mathscr{E}_{J}\right)\right)=0$.

(ii) Given $\mathscr{E}^{\prime}: \quad 0 \rightarrow J \rightarrow E^{\prime} \rightarrow B \rightarrow 0$ and $\mathscr{E}: 0 \rightarrow C_{J} \rightarrow E \rightarrow B \rightarrow 0$, then $\theta\left(\operatorname{cl}(\mathscr{E}), \operatorname{cl}\left(\mathscr{E}^{\prime}\right)\right)$ is the class of the extension

$$
\mathscr{E}^{\prime \prime}: \quad 0 \rightarrow J \rightarrow\left(E \times{ }_{B} E^{\prime}\right) / U \rightarrow B \rightarrow 0
$$

where $U$ is the ideal of pairs $(t,-t), t \in C_{J}$. 
To verify that $\theta$ is a group action (which we will not do anyway), it is easiest to construct $\theta$ in terms of the cotangent complex. Take a fixed presentation $B=P / I$ with $P$ a polynomial algebra. Given extensions $\mathscr{E}$ and $\mathscr{E}^{\prime}$ as above, there exist homomorphisms $\alpha: P \rightarrow E$ and $\alpha^{\prime}: P \rightarrow E^{\prime}$ (commuting with the projections to $B$ ). Then we get $\mathscr{E}^{\prime \prime}$ as $0 \rightarrow J \rightarrow E^{\prime \prime} \rightarrow B \rightarrow 0$, where

$$
E^{\prime \prime}=(P \oplus J) /\left\{\left(-x, \alpha x+\alpha^{\prime} x\right) \mid x \in I\right\} .
$$

The multiplication in $P \oplus J$ is defined as in (i), and straightforward checking shows that $\theta$ is a group action as desired.

4.2.7. Remark. $\theta$ reduces to addition in $T^{1}\left(B / A, C_{J}\right)$ if $J=C_{J}$, so that $J$ is just a $B$-module.

4.2.8. THEOREM. Let $A \rightarrow B$ be a ring homomorphism, $M$ a B-module and $e$ $\in T^{2}(B / A, M)$. Then there exists a pair $(J, \mu)$ as above, with $M \cong C_{J}$, such that $e$ is the obstruction $\mu^{*}\left(\mathrm{cl}\left(\mathscr{E}_{J}\right)\right)$.

Proof. Write $B=P / I$ with $P$ a polynomial algebra over $A$. By adjoining an extra variable to $P$ if necessary, we may assume that $(*)$ holds:

$$
\{t \in I \mid t I=(0)\}=(0) \text {. }
$$

Now it follows from the proof of 4.1.2 that $e=\operatorname{cl}(\mathscr{E})$, where

$$
\mathscr{E}: \quad 0 \longrightarrow M \stackrel{i}{\longrightarrow} J \stackrel{j}{\longrightarrow} P \longrightarrow B \longrightarrow 0 .
$$

$J$ is a commutative $A$-algebra, with multiplication $x \cdot y=j(x) \cdot y(x, y \in J)$, and by (*) one sees that $i$ induces $M \cong C_{J}$. Hence there is a homomorphism $\alpha: \mathscr{E} \rightarrow \mathscr{E}_{J}$, inducing some $\mu: B \rightarrow E_{J}$, such that $\alpha_{2}=$ id on $M$. By the first remark in 4.2.6, we have $e=\mu^{*}\left(\operatorname{cl}\left(\mathscr{E}_{J}\right)\right)$.

4.3. Infinitesimal deformations.

4.3.1. Let $A$ be a ring. Recall that an $A$-module $M$ is said to be flat over $A$ if the functor $N \rightarrow M \otimes_{A} N$ of $A$-modules is exact. In the applications (4.3.6) all flat modules will actually be free.

4.3.2. Definition. Let $A^{\prime}$ be a ring, $J$ a nilpotent ideal in $A^{\prime}$, and $B$ a flat $A=A^{\prime} / J$ algebra. By an (infinitesimal) deformation of $B$ over $A$ to $A^{\prime}$ we mean a flat $A^{\prime}$-algebra $B^{\prime}$ together with a homomorphism $B^{\prime} \rightarrow B$ inducing $B^{\prime} \mid J B^{\prime} \cong B$. In other words, a deformation is a commutative, "product," diagram

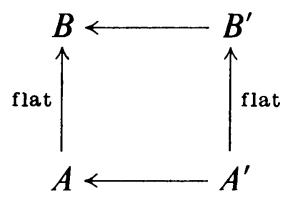

We denote by $\operatorname{Def}\left(B / A, A^{\prime}\right)$ the set of isomorphism classes of such diagrams. 
Let us now fix a diagram

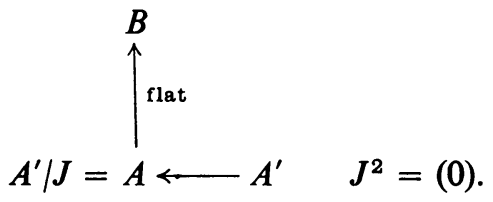

Note that in this case $J$ is an $A$-module.

From the sequence $A^{\prime} \rightarrow A \rightarrow B$ we get an exact sequence

$$
\begin{gathered}
0 \longrightarrow T^{1}\left(B / A, J \otimes_{A} B\right) \stackrel{u}{\longrightarrow} T^{1}\left(B / A^{\prime}, J \otimes_{A} B\right) \\
\stackrel{v}{\longrightarrow} T^{1}\left(A \mid A^{\prime}, J \otimes_{A} B\right) \stackrel{\partial}{\longrightarrow} T^{2}\left(B / A, J \otimes_{A} B\right) .
\end{gathered}
$$

Note that $T^{1}\left(A / A^{\prime}, J \otimes_{A} B\right)=\operatorname{Hom}_{B}\left(J \otimes_{A} B, J \otimes_{A} B\right)$ has a distinguished element $I$, corresponding to the identity endomorphism of $J \otimes_{A} B$ (or to the image of $\operatorname{cl}\left(A^{\prime}\right)$ in $\left.T^{1}\left(A / A^{\prime}, J\right) \rightarrow T^{1}\left(A / A^{\prime}, J \otimes_{A} B\right)\right)$.

4.3.3. Theorem. Let $A^{\prime}$ be a ring, $J$ an ideal in $A^{\prime}$ with $J^{2}=(0), B$ a flat $A=A^{\prime} \mid J-$ algebra, then

(i) $\operatorname{Def}\left(B / A, A^{\prime}\right) \neq \varnothing \Leftrightarrow$ the element $\partial(I)$ in $T^{2}\left(B / A, J \otimes_{A} B\right)$ is zero.

(ii) If $\partial(I)=0$, then $\operatorname{Def}\left(B / A, A^{\prime}\right)$ is a principal homogeneous space under $T^{1}\left(B / A, J \otimes_{A} B\right)$.

4.3.4. Infinitesimal automorphisms. If

$$
0 \longrightarrow M \stackrel{i}{\longrightarrow} E \stackrel{j}{\longrightarrow} B \longrightarrow 0
$$

is an extension of $B$ over $A$ by $M$, then the group of automorphisms of $E$ (leaving $M$ and $B$ fixed) is identified with $T^{0}(B / A, M)=\operatorname{Der}_{A}(B, M)$ by sending each derivation $\Delta$ to $\sigma: e \rightarrow e+\Delta(j(e))$.

In the diagram

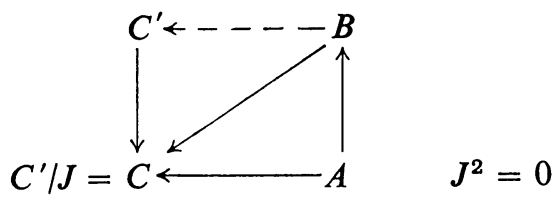

of 3.1.3, the obstruction to finding $B \rightarrow C^{\prime}$ is clearly $\operatorname{cl}\left(C^{\prime} \times_{C} B\right) \in T^{1}(B / A, J)$.

4.3.5. Remarks. One can define a kind of "Yoneda product" $e \cdot e^{\prime} \in T^{2}(B \mid A, B)$ for $e, e^{\prime}$ in $T^{1}(B / A, B)$, obtained from the ordinary Yoneda product $\left(e, e^{\prime}\right)$ for extensions of modules, by adding it to its transpose $\left(e^{\prime}, e\right)$. However, the obstruction to extending a deformation $B^{\prime}$ in $\operatorname{Def}\left(B / A, A[\varepsilon] / \varepsilon^{2}\right)$ to one in $\operatorname{Def}\left(B / A, A[\varepsilon] / \varepsilon^{3}\right)$ is an element $(e, e) \in T^{2}(B / A, B)$ satisfying $2(e, e)=e \cdot e\left(e=\operatorname{cl}\left(B^{\prime}\right)\right)$.

If $k$ is a perfect field, and $B$ is a reduced $k$-algebra of finite type, then it is easy to show that

$$
\operatorname{Ext}_{B}^{1}\left(\Omega_{B / k}, B\right) \cong T^{1}(B / k, B) .
$$

The Ext module is used in [4] by Grauert and Kerner to investigate deformations of reduced analytic spaces. 
4.3.6. Globalization. The definition of Def globalizes to give a set $\operatorname{Def}\left(X / S, S^{\prime}\right)$, where $X \rightarrow S$ is a flat morphism of schemes, and $S \rightarrow S^{\prime}$ is an infinitesimal immersion, defined by a nilpotent sheaf of ideals. Let $X$ be a scheme proper over $k$, and let $\Lambda$ be a complete noetherian local ring with residue field $k$. Let $C_{\Lambda}$ be the category of Artin local $\Lambda$-algebras, having the same residue field $k$. Then there is a complete local $\Lambda$-algebra $R$, and a formal prescheme $\mathscr{X}$ over $R$ which serves as a universal or "generic" deformation of $X$ (i.e., it "pro-represents" the functor $A$ $\rightarrow \operatorname{Def}(X / k, \operatorname{Spec} A)$ from $C_{\Lambda}$ to sets, in a certain weak sense). Computation of the cohomology of the sheaves $T^{i}\left(X \mid k, \mathcal{O}_{X}\right)$ yields information about $R$ (dimension, regularity, etc.). The material of 4.3 is discussed in more detail in [13].

5. Applications to the Riemann-Roch Theorem and duality. Using a projectivized version of the cotangent complex it is possible to restate the GrothendieckHirzebruch-Riemann-Roch Theorem so that it only depends on knowing the Todd class of a "relative object" and not on the Todd classes of absolute "tangent bundles." This should facilitate the generalization of the GHRR-Theorem to a situation where only the morphism is required to be well-behaved, and not the actual preschemes. (Throughout this section, we assume familiarity with the definitions and notations of [1].)

We start with some definitions. Let $Y$ be a noetherian prescheme, and $i$ a closed immersion of a prescheme $X$ into $P=P^{n}(Y)=Y \times_{\operatorname{spec} Z}$ Proj $Z\left[T_{0}, \ldots, T_{n}\right]$. Let $I$ be the sheaf of ideals on $P$ defining $X$. Then we have the following complex of sheaves of $O_{X}$-modules:

$$
I / I^{2} \stackrel{d}{\longrightarrow} i^{*} \Omega_{P / Y}
$$

Assume further that $X$ is actually Koszul over $Y$. (Under this hypothesis, the above complex, restricted to an affine situation: $\operatorname{Spec} B \rightarrow \operatorname{Spec} A$, is homotopic to any cotangent complex of $B$ over $A$.) Let $K(X)$ be the Grothendieck group of the category of coherent locally free sheaves on $X$. Since $X$ is locally a complete intersection in $P$ and $P$ is smooth over $Y, I / I^{2}$ and $i^{*} \Omega_{P / Y}$ are locally free sheaves on $X$. Let $L_{X / Y}$ be the class of $i^{*} \Omega_{P / Y}-I / I^{2}$ in $K(X)$. To justify the notation, we have the following

5.1.1. Proposition. $L_{X / Y}$ does not depend on the choice of the imbedding $i$.

Proof. (Note that under the hypotheses of the GHRR-Theorem ( $X$ and $Y$ regular and $Y$ quasi-projective), $K(X)$ is naturally isomorphic to the. Grothendieck group $K^{\prime}(X)$ of all coherent sheaves on $X$. In general, it is easy to see that the class of the image of $L_{X / Y}$ in $K^{\prime}(X)$ is exactly $\Omega_{X / Y}-T_{1}\left(X / Y, O_{X}\right)$, and so independent of the imbedding. When the natural map of $K(X)$ to $K^{\prime}(X)$ is an isomorphism, $L_{X / Y}$ is also independent of the imbedding.) We give the proof in the general case.

Let $i: X \rightarrow P$ and $i^{\prime}: X^{\prime} \rightarrow P^{\prime}$ be two imbeddings of $X$ into some projective space over $Y$. Since $f: X \rightarrow Y$ is a separated morphism, the map $i^{\prime \prime}: X \rightarrow P^{\prime \prime}=P \times_{Y} P^{\prime}$ 
defined by $i^{\prime \prime}=\left(i, i^{\prime}\right)$ is a closed immersion. Let $I$ (resp. $\left.I^{\prime \prime}\right)$ be the sheaf of ideals defining $X$ as a closed subscheme of $P$ (resp. $P^{\prime \prime}$ ). Let $\pi$ be the projection from $P^{\prime \prime}$ to $P$. From the triple $\left(P^{\prime \prime}, P, Y\right)$ we obtain the exact sequence

$$
0 \rightarrow \pi^{*} \Omega_{P / Y} \rightarrow \Omega_{P^{\prime \prime} / Y} \rightarrow \Omega_{P^{\prime \prime} / P} \rightarrow 0
$$

since $P^{\prime \prime}$ is smooth over $P$. Let $i$ (resp. $j$ ) be the closed immersion of $X$ in $P$ (resp. $\left.P^{\prime \prime}\right)$. Since $\Omega_{P^{\prime \prime} / P}$ is locally free, we have the exact sequence

$$
0 \rightarrow i^{*} \Omega_{P / Y} \rightarrow j^{*} \Omega_{P^{\prime \prime} / Y} \rightarrow j^{*} \Omega_{P^{\prime \prime} / P} \rightarrow 0
$$

of locally free sheaves of $O_{X}$-modules.

From the triangle $\left(X, P^{\prime \prime}, P\right)$ we get the exact sequence

$$
0 \rightarrow I / I^{2} \rightarrow J / J^{2} \rightarrow j^{*} \Omega_{P^{*} / P} \rightarrow 0
$$

of locally free sheaves of $O_{X}$-modules. Comparing the two exact sequences, we see that

$$
i^{*} \Omega_{P / Y}-I / I^{2}=j^{*} \Omega_{P^{\prime \prime} / Y}-J / J^{2}
$$

is true in $K^{\prime}(X)$. Repeating the above argument for $P^{\prime}$, we complete the proof.

5.1.2. Definition. Let $f: X \rightarrow Y$ be a morphism of preschemes. Then $f$ is strictly projective if $f$ can be factored into $X \rightarrow^{i} P \rightarrow^{\pi} Y$, where $i$ is a closed immersion, $P$ is some $P^{n}(Y)$ and $\pi$ is the natural projection.

5.1.3. Theorem. Let $f: X \rightarrow Y$ and $g: Y \rightarrow Z$ be strictly projective and Koszul morphisms of noetherian preschemes. Then $L_{X / Z}=L_{X / Z}+f^{\prime} L_{Y / Z}$, where $f^{\prime}$ is the map induced by $f^{*}$ from $K(Y)$ to $K(X)$.

Proof. Let $f$ (resp. $g$ ) factor into

$$
X \stackrel{i}{\longrightarrow} P \stackrel{\pi}{\longrightarrow} Y \quad\left(\text { resp. } Y \stackrel{j}{\longrightarrow} S \stackrel{\pi^{\prime}}{\longrightarrow} Z\right),
$$

where $P$ is some $\boldsymbol{P}^{n}(Y)$ and $S$ is some $\boldsymbol{P}^{n}(Z)$. Let $T=\boldsymbol{P}^{n}(S)$, so that $P=T \times{ }_{S} Y$. Then we have the following diagram:

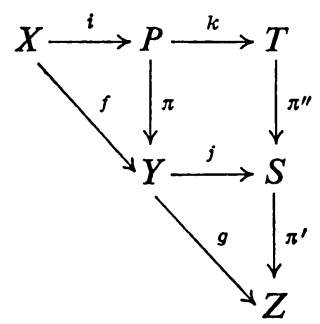

and $P$ is a locally complete intersection in $T$. Let $K$ (resp. $J$ ) (resp. $I$ ) be the sheaf of ideals defining $X$ (resp. $X$ ) (resp. $Y$ ) as a closed subscheme of $P$ (resp. $T$ ) (resp. $S$ ). Let $H$ be the sheaf of ideals defining $P$ in $T$. So $H / H^{2}=\pi^{*}\left(I / I^{2}\right)$. We obtain the following equations: 
(1) From the triple $(X, P, T): J / J^{2}=K / K^{2}+f^{\prime}\left(I / I^{2}\right)$ (in $\left.K(X)\right)$.

(2) From the triple $(T, S, Z): \Omega_{T / Z}=\Omega_{T / S}+\pi^{\prime \prime *} \Omega_{S / Z}$ (in $K(T)$ ).

(3) $\Omega_{R / Y}=K^{*} \Omega_{T / S}$.

We then conclude that:

$$
\begin{aligned}
L_{X / Z} & =-J / J^{2}+i^{*} k^{*} \Omega_{T / Z}=-K / K^{2}-f^{\prime}\left(I / I^{2}\right)+i^{*} \Omega_{P / Y}+f^{*} j^{*} \Omega_{S / Z} \\
& =\left(-K / K^{2}+i^{*} \Omega_{P / Y}-f^{\prime}\left(I / I^{2}-j^{*} \Omega_{S / Z}\right)\right)=L_{X / Y}+f^{\prime} L_{Y / Z} .
\end{aligned}
$$

5.1.4. Proposition. The Grothendieck-Hirzebruch-Riemann-Roch Theorem can be restated in the following form: Let $X$ and $Y$ be nonsingular quasi-projective algebraic varieties over an algebraically closed field $k, f$ a proper morphism from $X$ to $Y$. Then $\operatorname{ch} f_{!}(x)=f_{*}\left(\operatorname{ch} x_{\bullet} T\left(L_{X / Y}^{\prime}\right)\right)$, where $y^{\prime}$ is the dual of $y$.

Proof. The hypotheses imply that $f$ is strictly projective and Koszul, so $L_{X / Y}$ is defined. By Theorem 4.4.3 we have $\Omega_{X / K}=L_{X / Y}+f^{\prime}\left(\Omega_{Y / K}\right)$. (Since $X$ and $Y$ are smooth over $k$, it is easy to see that $L_{X / K}=\Omega_{X / K}$ and $L_{Y / K}=\Omega_{Y / K}$.) Recall that the GHRR-Theorem states that

(1) $f_{*}\left(\operatorname{ch} x_{\bullet} T(X)\right)=\operatorname{ch}\left(f_{:}(x)\right) . T(Y)$, where $x \in K(X), T(X)$ denotes the Todd class of the tangent bundle of $X$, and ch is the Chern character mapping $K(X)$ to $A(X)$.

By definition of the Todd class, it is an invertible element of the Chow ring, so we may rewrite (1) as

(2) $\operatorname{ch}\left(f_{!}(x)\right)=T(Y)^{-1} f_{*}\left(\operatorname{ch} x_{\bullet} T(X)\right)$.

Applying the relationship $x_{\bullet} f_{*}(y)=f_{*}\left(y_{\bullet} f^{*}(x)\right)$, (2) becomes

(3) $\operatorname{ch}\left(f_{!}(x)\right)=f_{*}\left(\operatorname{ch} x_{\bullet} T(X) . f^{*}\left(T(Y)^{-1}\right)\right)$.

Now $f^{*}$ is a ring homomorphism and commutes with forming Todd classes, so we get

(4) $\operatorname{ch}\left(f_{:}(x)\right)=f_{*}\left(\operatorname{ch} x_{\bullet} T(X) . T\left(f^{*}\left(\Omega_{Y}^{\prime}\right)^{-1}\right)\right)$.

Since the Todd class satisfies $T(x+y)=T(x) T(y)$, we obtain

(5) $\operatorname{ch}\left(f_{!}(x)\right)=f_{*}\left(\operatorname{ch} x_{\bullet} T\left(\Omega_{X / K}^{\prime}-f^{\prime} \Omega_{Y / K}^{\prime}\right)\right)$

and, therefore

(6) $\operatorname{ch}\left(f_{i}(x)\right)=f_{*}\left(\operatorname{ch} x_{\bullet} T\left(L_{X / Y}^{\prime}\right)\right)$.

It is clear that the above process is reversible, so we are done.

In particular, this form of the Riemann-Roch Theorem should be well adapted to the case of preschemes over a ring of algebraic integers, where the concept of "tangent bundle" no longer makes sense.

5.2.1. Recall that the operation $\Lambda$ which consists in raising a locally free sheaf of rank $r$ to its $r$ th exterior power induces a surjection from $K(X)$ to $\operatorname{Pic}(X)$, the group of isomorphism classes of invertible sheaves on $X$. This operation can be thought of as taking the first Chern class, and in fact is exactly that under the hypotheses of the GHRR-Theorem. Let $K_{X / Y} \in \operatorname{Pic}(X)$ be $\Lambda\left(L_{X / Y}\right)$, if $X$ is Koszul and strictly projective over $Y$, so that $L_{X / Y}$ is defined. Note that the hypotheses "Koszul and strictly projective" may be changed to "smooth," in which case $L_{X / Y}=$ the class of the locally free sheaf $\Omega_{X / Y}$. This canonical invertible sheaf on $X$ generalizes the sheaf of highest order differentials which appears in the Serre 
duality theorem. In fact, Grothendieck (see [6]) has shown the following: Let $k$ be a field, and $f$ a strictly projective Koszul morphism from $X$ to Spec $k$. Let $X$ be connected and $n$-dimensional. Let $F$ be a coherent sheaf on $X$. Then there exists a natural map from $\operatorname{Ext}^{n-q}\left(F, K_{X / Y}\right)$ to $\operatorname{Hom}_{K}\left(H^{q}(X, F), k\right)$ which is in fact an isomorphism. (Of course, Grothendieck has shown much more; his duality theorem does not require $Y$ to be the spectrum of a field. However, to give a concise statement of it requires the language of derived categories. (See [8, Chapter I].))

Note that even the very restricted form of the duality theorem which we have stated here is a significant generalization of the Serre duality theorem, since $X$ may be only a locally complete intersection and not nonsingular, and the field $k$ need not be perfect, let alone algebraically closed.

\title{
BIBLIOGRAPHY
}

1. A. Borel and J.-P. Serre, Le théorème de Riemann-Roch, Bull. Soc. Math. France 86 (1958), 97-136.

2. P. Cartier, Seminaire Cartan-Chevalley (1955-1956), Exposé XIII.

3. M. Gerstenhaber, On the deformation of rings and algebras, Ann. of Math. 79 (1964), 59-103.

4. H. Grauert and H. Kerner, Deformationen von singularitäten, komplexer Räume, Math. Ann. 1953 (1964), 236-260.

5. A. Grothendieck, Éléments de geometrie algebrique. I-IV, Institut des Hautes Études Scientifiques, $1960-1964$.

6. - Théorèmes de dualité pour les faisceaux algébriques cohérents, Seminaire Bourbaki, May 1957.

7. D. K. Harrison, Commutative algebras and cohomology, Trans. Amer. Math. Soc. 104 (1962), 191-204.

8. R. Hartshorne, Seminaire Hartshorne (The duality theorem of Grothendieck), Harvard Univ., Cambridge, Mass. (1963-1964).

9. E. Kunz, Differential Formen inseparabler algebraischer Funktionenkörper, Math. Z. 76 (1961), 56-74.

10. S. Lichtenbaum, Curves over discrete valuation rings, Ph.D. Thesis, Harvard Univ., Cambridge, Mass., 1964 (unpublished).

11. S. MacLane, Homology, Academic Press, New York, 1963.

12. Y. Nakai, On the theory of differentials in commutative rings, J. Math. Soc. Japan 13 (1961), 63-84.

13. M. Schlessinger, Infinitesimal deformations of singularities, Ph.D. Thesis, Harvard Univ., Cambridge, Mass., 1964 (unpublished).

14. J.-P. Serre, Algèbre locale-multiplicités, Mimeographed notes by P. Gabriel, Collège de France, 1958.

15. U. Shukla, Cohomologie des algèbres associatives, Ann. Sci. Ecole Norm. Sup. 78 (1961), 163-209.

16. M. Gerstenhaber, On the deformation of rings and algebras. II (unpublished preprint).

\author{
Princeton University, \\ Princeton, New Jersey \\ UNIVERSITY OF CALIFORNIA, \\ Berkeley, CALIForNia
}

